\title{
Hourglass stabilization and the virtual element method
}

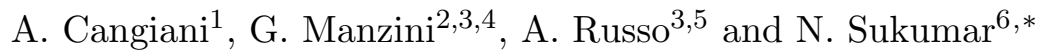 \\ 1 Department of Mathematics, University of Leicester, University Road - Leicester LE1 7RH, UK \\ 2 Theoretical Division, Los Alamos National Laboratory, Los Alamos, NM 87545, USA \\ ${ }^{3}$ Istituto di Matematica Applicata e Tecnologie Informatiche del CNR, via Ferrata 1, 27100 Pavia, Italy, \\ ${ }^{4}$ Centro per la Simulazione Numerica Avanzata, Istituto Universitario di Studi Superiori, 27100 Pavia, Italy \\ ${ }^{5}$ Dipartimento di Matematica e Applicazioni, Università di Milano-Bicocca, 20153 Milano, Italy \\ ${ }^{6}$ Department of Civil and Environmental Engineering, University of California, Davis, CA 95616, USA
}

\section{SUMMARY}

In this paper, we establish the connections between the virtual element method (VEM) and the hourglass control techniques that have been developed since the early 1980s to stabilize underintegrated $C^{0}$ Lagrange finite element methods. In the virtual element method, the bilinear form is decomposed into two parts: a consistent term that reproduces a given polynomial space and a correction term that provides stability. The essential ingredients of $\mathcal{C}^{0}$-continuous virtual element methods on polygonal and polyhedral meshes are described, which reveals that the variational approach adopted in the VEM affords a generalized and robust means to stabilize underintegrated finite elements. We focus on the heat conduction (Poisson) equation, and present a virtual element approach for the isoparametric four-node quadrilateral and the eight-node hexahedral element. In addition, we show quantitative

\footnotetext{
* Correspondence to: N. Sukumar, Department of Civil and Environmental Engineering, University of California,
} One Shields Avenue, Davis, CA 95616, USA. E-mail: nsukumar@ucdavis.edu 
comparisons of the consistency and stabilization matrices in the VEM to those in the hourglass control method of Belytschko and coworkers. Numerical examples in two and three dimensions are presented for different stabilization parameters, which reveals that the method satisfies the patch test, and delivers optimal rates of convergence in the $L^{2}$ norm and the $H^{1}$ seminorm for Poisson problems on quadrilateral, hexahedral, as well as arbitrary polygonal meshes. Copyright (c) 2014 John Wiley \& Sons, Ltd.

KEY WORDS: virtual element method, underintegration, hourglass control, consistency matrix, stabilization matrix, polygonal and polyhedral finite elements

\section{INTRODUCTION}

For linear and nonlinear transient analysis, underintegrated finite elements (FE) with hourglass stabilization is widely adopted. There are little benefits to using the full tensor-product Gauss quadrature $(2 \times 2$ for bilinear quadrilateral elements and $2 \times 2 \times 2$ for trilinear hexahedral elements): apart from the increased computational costs that are incurred, these elements are also prone to locking for incompressible materials for which some form of reduced integration is desirable. Hence, use of the one-point quadrature, combined with some form of stabilization, has been a topical area of research since the early 1980s. This feature is available in many of the finite element software codes [1-5].

Use of the one-point quadrature leads to a rank-deficient (singular) stiffness matrix and the presence of hourglass (solid continua) or spurious singular (Poisson equation) modes. This rank-deficiency is cured by the addition of a second matrix, the so-called hourglass stabilization matrix, which suppresses the spurious singular modes. Early studies to address this issue for low-order finite elements are due to Key [6] and Kosloff and Frazier [7]. To speed up

Copyright (C) 2014 John Wiley \& Sons, Ltd.

Int. J. Numer. Meth. Engng 2014; 1:1-33

Prepared using nmeauth.cls 
finite element computations, Flanagan and Belytschko [8] introduced the notion of orthogonal hourglass control, which was further extended and refined by them in subsequent studies [9-11]. In the literature, following Reference [8], many other initial contributions related to hourglass stabilization have also appeared [12-14]; for a historical perspective and an exhaustive list of references on this topic, the interested reader can see References [10, 15].

In this paper, we restrict our attention to the scalar diffusion (Poisson) problem, and draw connections with the formulations presented in References [8-10]. In keeping with the usage in the literature for problems in solid continua, we choose to use the term hourglass when referring to issues that pertain to the nonphysical modes of the stiffness (conductance) matrix. In Belytschko et al. [10], hourglass projection is obtained by using the consistency requirements and enforcing the elimination of the rank-deficiency of the discrete forms. Herein, we show that the recently proposed virtual element method [16], coined as VEM, can be viewed as a generalized hourglass stabilization technique for arbitrary-order polygonal and polyhedral finite element methods. In Section 4, the key properties of isoparametric finite elements in twoand three-dimensions are reviewed. In Section 5, we discuss reduced integration techniques for the Poisson problem recalling the well-known mean-gradient approach of Belytschko and coworkers [8-10]. The framework for first-order virtual elements is presented in Section 6. In Section 7, we show that virtual element discretizations can be constructed on general (convex and nonconvex) polygons and polyhedra and we provide a simple formula for the computation of the stiffness matrix. The derivation of the VEM for the bilinear isoparametric four-node quadrilateral element is presented in Section 8, and direct comparisons to the approach of Belytschko and coworkers [8-10] are made. The trilinear isoparametric eight-node hexahedral element is treated in Section 9, where quantitative links between the VEM and the hourglass 
control method in References $[8,10]$ are also established. In general, isoparametric eight-node hexahedral elements have curved faces and this paper provides an implementation for them. Numerical examples presented in Section 10 confirm that the VEM satisfies the patch test and achieves optimal rates of convergence in the $L^{2}$ norm and in the $H^{1}$ seminorm on quadrilateral, hexahedral, as well as polygonal meshes. We conclude with some final remarks in Section 11.

\section{NOTATION}

Let us summarize the main notations used in this paper. The symbol $d$ represents the spatial dimension, which is either 2 or 3 . Bold symbols like $\boldsymbol{s}, \boldsymbol{X}, \boldsymbol{K}$ represent vectors or matrices. A point $\boldsymbol{x} \in \mathbb{R}^{d}$ is denoted as $\boldsymbol{x} \equiv(x, y)$ in two-dimensions and as $\boldsymbol{x} \equiv(x, y, z)$ in three dimensions. The symbol $\Omega_{0}$ denotes either the biunit reference square $(-1,1)^{2}$ or the biunit reference cube $(-1,1)^{3}$, and $\Omega_{e}$ is the physical element, which is a quadrilateral or hexahedral finite element. The isoparametric four-node or eight-node finite element space on $\Omega_{e}$ is written as $\mathbb{Q}_{1}\left(\Omega_{e}\right)$. Note that hexahedra can have curved faces.

A general finite element is denoted by the letter $E$ and the finite element space attached to it is written as $V_{h}^{E}$. The number of vertices of $E$ is denoted by $n_{E}$, which is also the dimension of $V_{h}^{E}$ because the degrees of freedom of the finite elements considered in this paper are always associated with the vertices. The diameter of an element $E$ is denoted by $h_{E}$ and its area (or volume) by $|E|$; when $E=\Omega_{e}$, we use $h_{e}$ instead of $h_{\Omega_{e}}$. The space of polynomials of degree less than or equal to $k$ on $E$ is denoted by $\mathcal{P}_{k}(E)$.

Copyright (C) 2014 John Wiley \& Sons, Ltd.

Int. J. Numer. Meth. Engng 2014; 1:1-33

Prepared using nmeauth.cls 


\section{THE POISSON PROBLEM}

For the sake of simplicity, we consider the following Poisson problem with nonhomogeneous Dirichlet boundary conditions:

$$
\begin{gathered}
-\Delta u=f \quad \text { in } \Omega \subset \mathbb{R}^{d} \\
u=g \quad \text { on } \partial \Omega,
\end{gathered}
$$

where $d=2,3$ is the spatial dimension and $f$ is a forcing function. The bilinear form associated with problem (1) is denoted by $a(\cdot, \cdot)$ :

$$
a(u, v):=\int_{\Omega} \nabla u \cdot \nabla v \mathrm{~d} \boldsymbol{x} .
$$

If $E \subset \Omega$ is a generic finite element, we denote by $a^{E}(\cdot, \cdot)$ the restriction of $a(\cdot, \cdot)$ to $E$; that is,

$$
a^{E}(u, v):=\int_{E} \nabla u \cdot \nabla v \mathrm{~d} \boldsymbol{x}
$$

with $\sum_{E} a^{E}(u, v)=a(u, v)$.

The numerical approximation of $a^{E}(\cdot, \cdot)$ is denoted by $a_{h}^{E}(\cdot, \cdot)$, and the corresponding global approximation $a_{h}(\cdot, \cdot)$ of $a(\cdot, \cdot)$ is defined as

$$
a_{h}(u, v):=\sum_{E} a_{h}^{E}(u, v)
$$

If $V_{h}^{E}$ is the finite element space attached to $E$ and $\left\{\phi_{1}, \ldots \phi_{n_{E}}\right\}$ are the Lagrange shape functions, the exact $n_{E} \times n_{E}$ element stiffness matrix $\boldsymbol{K}^{E}$ is given by

$$
\left(\boldsymbol{K}^{E}\right)_{i j}:=a^{E}\left(\phi_{i}, \phi_{j}\right):=\int_{E} \nabla \phi_{i} \cdot \nabla \phi_{j} \mathrm{~d} \boldsymbol{x} \quad i, j=1, \ldots, n_{E} .
$$

The stiffness matrix corresponding to $a_{h}^{E}(\cdot, \cdot)$ is defined as

$$
\left(\boldsymbol{K}_{h}^{E}\right)_{i j}:=a_{h}^{E}\left(\phi_{i}, \phi_{j}\right) \quad i, j=1, \ldots, n_{E} .
$$

The superscript $E$ and the subscript $h$ will be often omitted when no confusion can arise.

Copyright (C) 2014 John Wiley \& Sons, Ltd.

Int. J. Numer. Meth. Engng 2014; 1:1-33

Prepared using nmeauth.cls 


\subsection{Linear consistency and stability}

We consider spaces $V_{h}^{E}$ that contain linear polynomials and other functions that are not linear.

In order to guarantee stable and convergent approximations, we say that $a_{h}^{E}(\cdot, \cdot)$ is

(i) linearly consistent if it is exact when one of the two entries is a linear polynomial:

$$
a_{h}^{E}\left(u_{h}, p_{1}\right)=a^{E}\left(u_{h}, p_{1}\right) \quad \text { for all } u_{h} \in V_{h}^{E}, p_{1} \in \mathcal{P}_{1}(E)
$$

(ii) stable if it is uniformly bounded from above and below by the exact bilinear form $a^{E}(\cdot, \cdot):$

there exist two constants $\alpha_{*}$ and $\alpha^{*}$ independent of $h$ such that

$$
\alpha_{*} a^{E}\left(v_{h}, v_{h}\right) \leq a_{h}^{E}\left(v_{h}, v_{h}\right) \leq \alpha^{*} a^{E}\left(v_{h}, v_{h}\right) \quad \text { for all } v_{h} \in V_{h}^{E} .
$$

The two properties defined above guarantees that the method passes the patch test, which we state as a Theorem for future reference in this paper.

Theorem 1. Let the approximate bilinear form $a_{h}^{E}(\cdot, \cdot)$ be linearly consistent (3) and stable (4). Then, the corresponding Galerkin method passes the linear patch test.

\section{LOW-ORDER ISOPARAMETRIC ELEMENTS IN 2D AND 3D}

Let us recall the essential properties of the four-node quadrilateral and the eight-node hexahedral isoparametric finite elements.

\subsection{Quadrilateral element}

Let $\Omega_{e}$ be a convex quadrilateral with vertices $\boldsymbol{x}_{i}=\left(x_{i}, y_{i}\right), i=1, \ldots, 4$, and $\Omega_{0}=(-1,1)^{2}$ be the reference biunit square. Its vertices $\boldsymbol{\xi}_{i}=\left(\xi_{i}, \eta_{i}\right), i=1, \ldots, 4$, are ordered as follows:

$$
\boldsymbol{\xi}_{1}=(-1,-1), \quad \boldsymbol{\xi}_{2}=(+1,-1), \quad \boldsymbol{\xi}_{3}=(+1,+1), \quad \boldsymbol{\xi}_{4}=(-1,+1) .
$$

Copyright (c) 2014 John Wiley \& Sons, Ltd.

Int. J. Numer. Meth. Engng 2014; 1:1-33

Prepared using nmeauth.cls 
The bilinear isoparametric shape functions $N_{1}, \ldots, N_{4}$ on the reference square $\Omega_{0}$ are defined
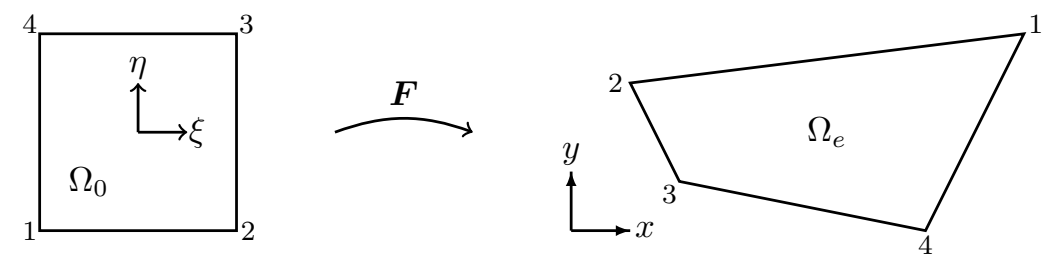

Figure 1. Reference biunit square and a mapped convex quadrilateral in physical space.

by

$$
N_{i}(\xi, \eta):=\frac{1}{4}\left(1+\xi \xi_{i}\right)\left(1+\eta \eta_{i}\right)
$$

so that $N_{i}\left(\boldsymbol{\xi}_{j}\right)=\delta_{i j}$. The bilinear transformation $\boldsymbol{F}: \mathbb{R}^{2} \longrightarrow \mathbb{R}^{2}$ that maps the reference square $\Omega_{0}$ to the physical element $\Omega_{e}$ (see Figure 1) is given by:

$$
\boldsymbol{x}=\boldsymbol{F}(\boldsymbol{\xi}):=\sum_{i=1}^{4} N_{i}(\boldsymbol{\xi}) \boldsymbol{x}_{i} .
$$

If the quadrilateral $\Omega_{e}$ is convex, the map $\boldsymbol{F}$ is nonsingular on $\Omega_{0}$; that is,

$$
\operatorname{det}\left(\boldsymbol{J}_{\boldsymbol{F}}(\boldsymbol{\xi})\right)>0 \quad \text { for } \boldsymbol{\xi} \in \Omega_{0},
$$

where $\boldsymbol{J}_{\boldsymbol{F}}$ is the Jacobian matrix of $\boldsymbol{F}$. The isoparametric finite element space $\mathbb{Q}_{1}\left(\Omega_{0}\right)$ on the reference element contains all bilinear functions in $\xi$ and $\eta$ and can be defined by

$$
\mathbb{Q}_{1}\left(\Omega_{0}\right):=\operatorname{span}\left\{N_{1}, \ldots, N_{4}\right\}
$$

The corresponding finite element space $\mathbb{Q}_{1}\left(\Omega_{e}\right)$ on the physical element is defined by

$$
\mathbb{Q}_{1}\left(\Omega_{e}\right):=\left\{v: \Omega_{e} \longrightarrow \mathbb{R} \text { such that } v \circ \boldsymbol{F} \in \mathbb{Q}_{1}\left(\Omega_{0}\right)\right\}
$$

The standard basis for $\mathbb{Q}_{1}\left(\Omega_{e}\right)$ is given by $\left\{\phi_{1}, \ldots, \phi_{4}\right\}$, where

$$
\phi_{i}:=N_{i} \circ \boldsymbol{F}^{-1}
$$

Copyright (C) 2014 John Wiley \& Sons, Ltd.

Int. J. Numer. Meth. Engng 2014; 1:1-33

Prepared using nmeauth.cls 
and we have $\phi_{i}\left(\boldsymbol{x}_{j}\right)=\delta_{i j}$. From (5) and (7), we note that the space $\mathbb{Q}_{1}\left(\Omega_{e}\right)$ contains all linear polynomials; hence, $\mathbb{Q}_{1}\left(\Omega_{e}\right)$ is generated by $\{1, x, y\}$ and a fourth function that is not linear. For instance, if $\Omega_{e}=\Omega_{0}$,

$$
\mathbb{Q}_{1}\left(\Omega_{e}\right)=\operatorname{span}\{1, x, y, x y\}
$$

\subsection{Hexahedral element}

The definitions for the trilinear hexahedral element closely follow those indicated for the bilinear quadrilateral element. The vertices of the hexahedral element $\Omega_{e}$ in the physical coordinate system are denoted by $\boldsymbol{x}_{i}=\left(x_{i}, y_{i}, z_{i}\right), i=1, \ldots, 8$, and the reference cube is $\Omega_{0}=(-1,1)^{3}$. The vertices $\boldsymbol{\xi}_{i}=\left(\xi_{i}, \eta_{i}, \zeta_{i}\right), i=1, \ldots, 8$, in the reference cube are ordered as follows:

$$
\begin{aligned}
& \boldsymbol{\xi}_{1}=(-1,-1,-1), \boldsymbol{\xi}_{2}=(+1,-1,-1), \boldsymbol{\xi}_{3}=(+1,+1,-1), \boldsymbol{\xi}_{4}=(-1,+1,-1), \\
& \boldsymbol{\xi}_{5}=(-1,-1,+1), \boldsymbol{\xi}_{6}=(+1,-1,+1), \boldsymbol{\xi}_{7}=(+1,+1,+1), \boldsymbol{\xi}_{8}=(-1,+1,+1) .
\end{aligned}
$$

The trilinear isoparametric shape functions $N_{1}, \ldots, N_{8}$ on the reference cube $\Omega_{0}$ are defined by

$$
N_{i}(\xi, \eta, \zeta):=\frac{1}{8}\left(1+\xi \xi_{i}\right)\left(1+\eta \eta_{i}\right)\left(1+\zeta \zeta_{i}\right)
$$

so that $N_{i}\left(\boldsymbol{\xi}_{j}\right)=\delta_{i j}$. The trilinear transformation $\boldsymbol{F}: \mathbb{R}^{3} \longrightarrow \mathbb{R}^{3}$ that maps the reference cube $\Omega_{0}$ to the hexahedral element $\Omega_{e}$ (see Figure 2) is given by:

$$
\boldsymbol{x}=\boldsymbol{F}(\boldsymbol{\xi}):=\sum_{i=1}^{8} N_{i}(\boldsymbol{\xi}) \boldsymbol{x}_{i} .
$$

We assume that the map $\boldsymbol{F}$ is nonsingular on $\Omega_{0}$; that is,

$$
\operatorname{det}\left(\boldsymbol{J}_{\boldsymbol{F}}(\boldsymbol{\xi})\right)>0 \quad \text { for } \boldsymbol{\xi} \in \Omega_{0} .
$$

Copyright (C) 2014 John Wiley \& Sons, Ltd.

Int. J. Numer. Meth. Engng 2014; 1:1-33

Prepared using nmeauth.cls 

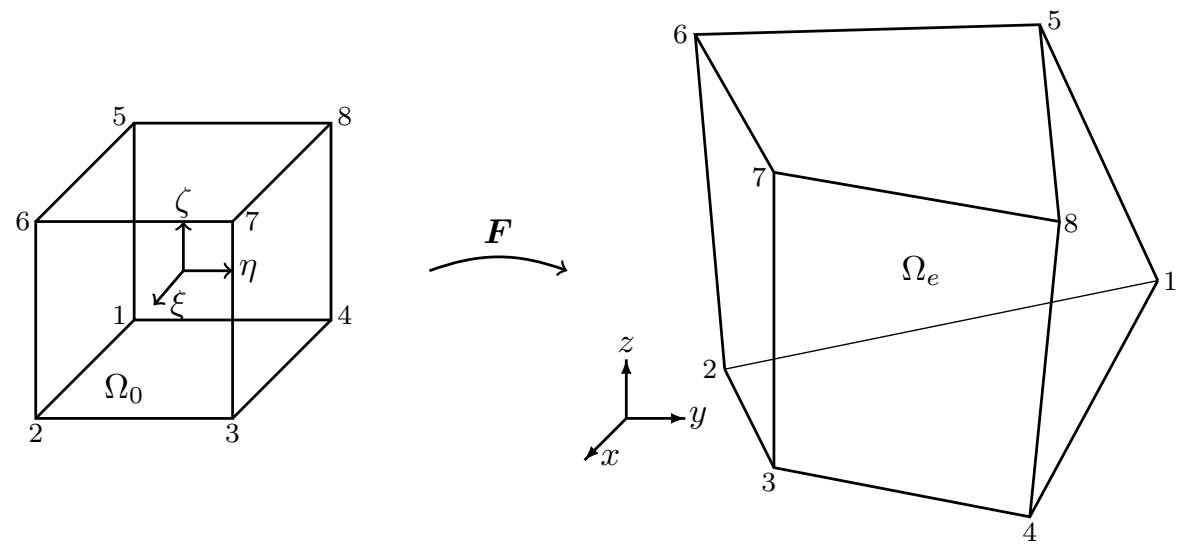

Figure 2. Reference biunit cube and a mapped hexahedron in physical space. In general, $\Omega_{e}$ has curved faces.

The isoparametric finite element space $\mathbb{Q}_{1}\left(\Omega_{0}\right)$ on the reference element contains trilinear functions in $\xi, \eta$ and $\zeta$, and can be defined as

$$
\mathbb{Q}_{1}\left(\Omega_{0}\right)=\operatorname{span}\left\{N_{1}, \ldots, N_{8}\right\}
$$

The corresponding finite element space $\mathbb{Q}_{1}\left(\Omega_{e}\right)$ on the physical element is defined by

$$
\mathbb{Q}_{1}\left(\Omega_{e}\right)=\left\{v: \Omega_{e} \longrightarrow \mathbb{R} \text { such that } v \circ \boldsymbol{F} \in \mathbb{Q}_{1}\left(\Omega_{0}\right)\right\}
$$

The standard basis for $\mathbb{Q}_{1}\left(\Omega_{e}\right)$ is given by $\left\{\phi_{1}, \ldots, \phi_{8}\right\}$, where

$$
\phi_{i}:=N_{i} \circ \boldsymbol{F}^{-1}
$$

and we have $\phi_{i}\left(\boldsymbol{x}_{j}\right)=\delta_{i j}$. We point out that the space $\mathbb{Q}_{1}\left(\Omega_{e}\right)$ is generated by $\{1, x, y, z\}$ and four more functions that are not linear. For instance, if $\Omega_{e}=\Omega_{0}$,

$$
\mathbb{Q}_{1}\left(\Omega_{e}\right)=\operatorname{span}\{1, x, y, z, x y, x z, y z, x y z\}
$$

Copyright (c) 2014 John Wiley \& Sons, Ltd.

Int. J. Numer. Meth. Engng 2014; 1:1-33

Prepared using nmeauth.cls 


\section{REDUCED INTEGRATION TECHNIQUES FOR THE POISSON PROBLEM}

Now, we consider the isoparametric finite element approximation of problem (1). The domain $\Omega$ is partitioned into a finite number of isoparametric elements $\Omega_{e}$, and let $\mathbb{Q}_{1}\left(\Omega_{e}\right)$ be the corresponding isoparametric finite element space introduced in the previous section. The element stiffness matrix, is given by

$$
\boldsymbol{K}_{i j}=\int_{\Omega_{e}} \nabla \phi_{i} \cdot \nabla \phi_{j} \mathrm{~d} \boldsymbol{x} \quad i, j=1, \ldots, 2^{d},
$$

which can be computed by mapping back to the reference element and then approximating the integral with a quadrature formula. In Appendix A, we present the requirements on a quadrature rule to meet the linear consistency property.

One-point quadrature approaches to compute the element stiffness matrix are based on the simple approximation of (9) given by

$$
\left|\Omega_{e}\right| \overline{\nabla \phi_{i}} \cdot \overline{\nabla \phi_{j}} .
$$

Concerning the definition of $\overline{\nabla \phi_{i}}$, there are a few options that are available. However, not all choices maintain linear consistency that (10) is designed to achieve.

Irrespective of how a mean-gradient is defined, the element stiffness matrix $\boldsymbol{K}$ is the product of two matrices each with rank $d$; hence, $\boldsymbol{K}$ has rank (at most) $d$ and is rank-deficient. Because the constant vector lies in the kernel of $\boldsymbol{K}$, the correct rank of the element stiffness matrix is 3 for quadrilateral and 7 for hexahedral elements. The rank-deficiency of $\boldsymbol{K}$ allows spurious singular modes to be present that can pollute the numerical solution. This deficiency is cured by adding a correction term (stabilization matrix) to (10), and the various remedies for underintegrated finite elements fall under the umbrella of what has come to be known as hourglass control methods. In what follows, each method that we consider will be characterized by the 
decomposition

$$
\boldsymbol{K} \approx \boldsymbol{K}_{\mathrm{C}}+\boldsymbol{K}_{\mathrm{s}}
$$

where $\boldsymbol{K}_{\mathrm{c}}$ is an instance of (10) (consistency term) and $\boldsymbol{K}_{\mathrm{s}}$ is an hourglass correction (stability term).

The mean-gradient approach of Flanagan and Belytschko [8] is based on defining

$$
\overline{\nabla \phi_{i}}:=\frac{1}{\left|\Omega_{e}\right|} \int_{\Omega_{e}} \nabla \phi_{i} \mathrm{~d} \boldsymbol{x}
$$

Following References [8,10], we introduce the $d \times 2^{d}$ matrix $\boldsymbol{B}$ whose columns are the meangradients of the shape functions $\phi_{i}$, and write $\boldsymbol{B}$ as

$$
\boldsymbol{B}=\left[\begin{array}{c}
\boldsymbol{b}_{1}^{\mathrm{T}} \\
\vdots \\
\boldsymbol{b}_{d}^{\mathrm{T}}
\end{array}\right],
$$

where

$$
\boldsymbol{b}_{1}^{\mathrm{T}}=\frac{1}{\left|\Omega_{e}\right|}\left[\int_{\Omega_{e}} \frac{\partial \phi_{1}}{\partial x} \mathrm{~d} \boldsymbol{x} \quad \int_{\Omega_{e}} \frac{\partial \phi_{2}}{\partial x} \mathrm{~d} \boldsymbol{x} \quad \cdots \quad \int_{\Omega_{e}} \frac{\partial \phi_{2^{d}}}{\partial x} \mathrm{~d} \boldsymbol{x}\right],
$$

and so on. With the above notation at hand, the consistency matrix due to Flanagan and Belytschko [8] reads:

$$
\boldsymbol{K}_{\mathrm{C}}^{\mathrm{FB}}:=\left|\Omega_{e}\right| \sum_{\delta=1}^{d} \boldsymbol{b}_{\delta} \boldsymbol{b}_{\delta}^{\mathrm{T}} .
$$

On observing that $\overline{\nabla p_{1}}=\nabla p_{1}$, because $\nabla p_{1}$ is a constant vector, we have

$$
\begin{aligned}
a_{c}^{\mathrm{FB}}\left(v_{h}, p_{1}\right) & =\left|\Omega_{e}\right| \overline{\nabla v_{h}} \cdot \overline{\nabla p_{1}}=\left(\int_{\Omega_{e}} \nabla v_{h} \mathrm{~d} \boldsymbol{x}\right) \cdot \nabla p_{1}=\int_{\Omega_{e}} \nabla v_{h} \cdot \nabla p_{1} \mathrm{~d} \boldsymbol{x} \\
& =a^{e}\left(v_{h}, p_{1}\right),
\end{aligned}
$$

which shows that the approximate bilinear form corresponding to (13) is linearly consistent. Another classical method, which is referred to as the Hallquist-approach $[3,17]$ is that of using Copyright (C) 2014 John Wiley \& Sons, Ltd. Int. J. Numer. Meth. Engng 2014; 1:1-33 Prepared using nmeauth.cls 
the one-point Gauss quadrature for $\overline{\nabla \phi_{i}}$; that is,

$$
\overline{\nabla \phi_{i}}:=\nabla \phi_{i}(\overline{\boldsymbol{x}})
$$

where $\overline{\boldsymbol{x}}:=\frac{1}{2^{d}} \sum_{i=1}^{2^{d}} \boldsymbol{x}_{i}$ is the centroid of the vertices of $\Omega_{e}$. In two dimensions, the meangradient formula and the one-point Gauss formula coincide; that is,

$$
\frac{1}{\left|\Omega_{e}\right|} \int_{\Omega_{e}} \nabla \phi_{i} \mathrm{~d} \boldsymbol{x}=\nabla \phi_{i}(\overline{\boldsymbol{x}}) \quad(d=2 \text { only })
$$

A proof of this fact is given in Section 8. On the other hand, for a hexahedral element the mean-gradient and the gradient obtained via the one-point Gauss rule do not coincide, and employing (14) does not lead to a linearly consistent scheme, which was recognized in Reference [8]. We now discuss the hourglass control method of Flanagan and Belytschko [8]. The two- and three-dimensional cases are treated separately, and we begin with the twodimensional case.

\subsection{Two-dimensional case}

For $d=2$, there is one hourglass vector, namely

$$
\boldsymbol{\Gamma}=\left[\begin{array}{llll}
+1 & -1 & +1 & -1
\end{array}\right]^{\mathrm{T}}
$$

such that $\boldsymbol{K}_{\mathrm{C}}^{\mathrm{FB}} \boldsymbol{\Gamma}=0$. In what follows, we shall also make use of the vectors

$$
\boldsymbol{s}:=\left[\begin{array}{llll}
+1 & +1 & +1 & +1
\end{array}\right]^{\mathrm{T}}, \boldsymbol{X}:=\left[\begin{array}{llll}
x_{1} & x_{2} & x_{3} & x_{4}
\end{array}\right]^{\mathrm{T}}, \boldsymbol{Y}:=\left[\begin{array}{llll}
y_{1} & y_{2} & y_{3} & y_{4}
\end{array}\right]^{\mathrm{T}},
$$

which are associated with the linear polynomial basis $\{1, x, y\}$. Direct verification yields the orthogonality relations:

$$
\boldsymbol{s}^{\mathrm{T}} \boldsymbol{\Gamma}=0, \quad \boldsymbol{b}_{\delta}^{\mathrm{T}} \boldsymbol{s}=0, \quad \boldsymbol{b}_{\delta}^{\mathrm{T}} \boldsymbol{\Gamma}=0 \quad \text { for } \delta=1,2,
$$

Copyright (c) 2014 John Wiley \& Sons, Ltd.

Int. J. Numer. Meth. Engng 2014; 1:1-33

Prepared using nmeauth.cls 
so that every vector $\gamma \in \mathbb{R}^{4}$ can be expanded as $\gamma=a_{1} \boldsymbol{b}_{1}+a_{2} \boldsymbol{b}_{2}+a_{3} s+a_{4} \boldsymbol{\Gamma}$. Because $\boldsymbol{b}_{1}^{\mathrm{T}} \boldsymbol{X}=\boldsymbol{b}_{2}^{\mathrm{T}} \boldsymbol{Y}=1$ and $\boldsymbol{b}_{1}^{\mathrm{T}} \boldsymbol{Y}=\boldsymbol{b}_{2}^{\mathrm{T}} \boldsymbol{X}=0$, imposing orthogonality of $\boldsymbol{\gamma}$ with respect to the affine basis in (17) yields the one-dimensional vector space spanned by the vector $[8,10]$ :

$$
\boldsymbol{\gamma}=\boldsymbol{\Gamma}-\left(\boldsymbol{\Gamma}^{\mathrm{T}} \boldsymbol{X}\right) \boldsymbol{b}_{1}-\left(\boldsymbol{\Gamma}^{\mathrm{T}} \boldsymbol{Y}\right) \boldsymbol{b}_{2}
$$

The vector $\gamma$ resulting from (18) is explicitly given by Flanagan and Belytschko [8, p. 690]:

$$
\gamma=\frac{1}{4\left|\Omega_{e}\right|}\left[\begin{array}{l}
x_{2}\left(y_{3}-y_{4}\right)+x_{3}\left(y_{4}-y_{2}\right)+x_{4}\left(y_{2}-y_{3}\right) \\
x_{3}\left(y_{1}-y_{4}\right)+x_{4}\left(y_{3}-y_{1}\right)+x_{1}\left(y_{4}-y_{3}\right) \\
x_{4}\left(y_{1}-y_{2}\right)+x_{1}\left(y_{2}-y_{4}\right)+x_{2}\left(y_{4}-y_{1}\right) \\
x_{1}\left(y_{3}-y_{2}\right)+x_{2}\left(y_{1}-y_{3}\right)+x_{3}\left(y_{2}-y_{1}\right)
\end{array}\right] .
$$

We can now define the stability term:

$$
\boldsymbol{K}_{\mathrm{s}}^{\mathrm{FB}}:=\left|\Omega_{e}\right| \bar{\epsilon} \boldsymbol{\gamma} \gamma^{\mathrm{T}} \quad(d=2),
$$

with $\bar{\epsilon} \in \mathbb{R}$ being a parameter that reflects the freedom of choice for the vector $\gamma$. In order to ensure convergence, the parameter $\bar{\epsilon}$ must be correctly scaled $\left(\bar{\epsilon}=\mathcal{O}\left(h_{e}^{-2}\right)\right.$ when $\left.d=2\right)$. This fact is clearly established via the VEM in Section 7.

By construction, $\boldsymbol{K}_{\mathrm{s}}^{\mathrm{FB}} \boldsymbol{v}=0$ whenever $\boldsymbol{v}$ represents an affine field, that is, a linear combination of the vectors defined in (17), thus ensuring that the method given by

$$
\boldsymbol{K}^{\mathrm{FB}}:=\boldsymbol{K}_{\mathrm{C}}^{\mathrm{FB}}+\boldsymbol{K}_{\mathrm{s}}^{\mathrm{FB}}
$$

is linearly consistent. In addition, $\boldsymbol{K}^{\mathrm{FB}}$ has the correct rank, thus excluding the presence of spurious singular modes. Hence, by Theorem 1 the corresponding method passes the patch test.

Copyright (C) 2014 John Wiley \& Sons, Ltd.

Int. J. Numer. Meth. Engng 2014; 1:1-33

Prepared using nmeauth.cls 


\subsection{Three-dimensional case}

For $d=3$, we proceed as in the $d=2$ case. Here, the spurious modes are related to the four vectors $[8]$

$$
\begin{array}{ll}
\boldsymbol{\Gamma}_{1}=[+1+1-1-1-1-1+1+1]^{\mathrm{T}}, & \boldsymbol{\Gamma}_{2}=[+1-1-1+1-1+1+1-1]^{\mathrm{T}}, \\
\boldsymbol{\Gamma}_{3}=[+1-1+1-1+1-1+1-1]^{\mathrm{T}}, & \boldsymbol{\Gamma}_{4}=[-1+1-1+1+1-1+1-1]^{\mathrm{T}},
\end{array}
$$

which represent the hourglass modes of the reference element. In analogy with the twodimensional case, we define the vectors:

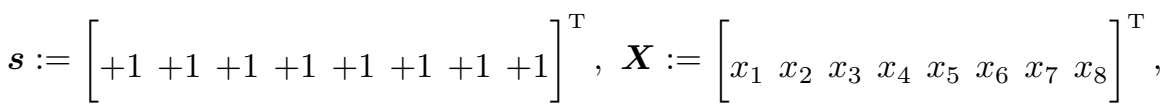

$$
\begin{aligned}
& \boldsymbol{Y}:=\left[\begin{array}{llllllll}
y_{1} & y_{2} & y_{3} & y_{4} & y_{5} & y_{6} & y_{7} & y_{8}
\end{array}\right]^{\mathrm{T}}, \boldsymbol{Z}:=\left[\begin{array}{llllllll}
z_{1} & z_{2} & z_{3} & z_{4} & z_{5} & z_{6} & z_{7} & z_{8}
\end{array}\right]^{\mathrm{T}} .
\end{aligned}
$$

Flanagan and Belytschko [8] infer that the eight vectors $\boldsymbol{\Gamma}_{\alpha}, \boldsymbol{b}_{\delta}$, and $s$ are linearly independent and form a basis for $\mathbb{R}^{8}$, and hence consider the following four vectors $\gamma_{\alpha} \in \mathbb{R}^{8}$ :

$$
\gamma_{\alpha}:=a_{\alpha} s+\sum_{\delta=1}^{3} a_{\alpha \delta} \boldsymbol{b}_{\delta}+\sum_{\beta=1}^{4} \tilde{a}_{\alpha \beta} \boldsymbol{\Gamma}_{\beta}, \quad \alpha=1, \ldots, 4
$$

that are mutually linearly independent and orthogonal to all vectors associated with affine fields. On imposing orthogonality with affine fields, the four basis vectors are obtained:

$$
\gamma_{\alpha}=\boldsymbol{\Gamma}_{\alpha}-\left(\boldsymbol{\Gamma}_{\alpha}^{\mathrm{T}} \boldsymbol{X}\right) \boldsymbol{b}_{1}-\left(\boldsymbol{\Gamma}_{\alpha}^{\mathrm{T}} \boldsymbol{Y}\right) \boldsymbol{b}_{2}-\left(\boldsymbol{\Gamma}_{\alpha}^{\mathrm{T}} \boldsymbol{Z}\right) \boldsymbol{b}_{3}, \quad \alpha=1, \ldots, 4
$$

Since each of the vectors $\gamma_{\alpha}$ is a linear combination of just one of the hourglass vectors and the three $\boldsymbol{b}_{\delta}$ vectors, and all these vectors are linearly independent, it follows that the $\gamma_{\alpha}$ are linearly independent as required. Hence,

$$
\boldsymbol{K}_{\mathrm{S}}^{\mathrm{FB}}:=\left|\Omega_{e}\right| \sum_{\alpha=1}^{4} \bar{\epsilon}_{\alpha} \gamma_{\alpha} \gamma_{\alpha}^{\mathrm{T}} \quad(d=3),
$$

Copyright (C) 2014 John Wiley \& Sons, Ltd.

Int. J. Numer. Meth. Engng 2014; 1:1-33

Prepared using nmeauth.cls 
and $\bar{\epsilon}_{\alpha}:=\bar{\epsilon} \in \mathbb{R}$ (free parameter) for all $\alpha$ is the choice made in Reference [8]. Finally, the element stiffness matrix $\boldsymbol{K}^{\mathrm{FB}}$ can be constructed as in (21):

$$
\boldsymbol{K}^{\mathrm{FB}}:=\boldsymbol{K}_{\mathrm{C}}^{\mathrm{FB}}+\boldsymbol{K}_{\mathrm{s}}^{\mathrm{FB}}=\left|\Omega_{e}\right|\left(\sum_{i=1}^{3} \boldsymbol{b}_{i} \boldsymbol{b}_{i}^{\mathrm{T}}+\bar{\epsilon} \sum_{\alpha=1}^{4} \gamma_{\alpha} \gamma_{\alpha}^{\mathrm{T}}\right),
$$

which leads to a linearly consistent and stable method that passes the patch test.

\section{VIRTUAL ELEMENT FRAMEWORK}

The virtual element method is a general framework for devising consistent and stable Galerkin approximations in the sense of Section 3.1. We assume that the space $V_{h}^{E}$ contains linear polynomials and other functions that are not linear. The key steps of the VEM are as follows:

- a definition of the space $V_{h}^{E}$ and vertex degrees of freedom that are associated with the Lagrange shape functions $\left\{\phi_{1}, \ldots, \phi_{n_{E}}\right\}$.

- the construction of a projector

$$
\Pi_{E}^{\nabla}: V_{h}^{E} \longrightarrow \mathcal{P}_{1}(E)
$$

which is orthogonal with respect to the $a^{E}(\cdot, \cdot)$ scalar product that is induced by problem (1).

- the exact decomposition of the local stiffness matrix (9) as the sum of a computable consistency matrix $\boldsymbol{K}_{\mathrm{C}}^{\mathrm{VEM}}$ and an uncomputable stability matrix $\boldsymbol{K}_{\mathrm{S}}^{*}$ as

$$
\boldsymbol{K}^{E}=\boldsymbol{K}_{\mathrm{C}}^{\mathrm{VEM}}+\boldsymbol{K}_{\mathrm{s}}^{*}
$$

with

$$
\begin{aligned}
\left(\boldsymbol{K}_{\mathrm{C}}^{\mathrm{VEM}}\right)_{i j} & :=\int_{E} \nabla \Pi_{E}^{\nabla} \phi_{i} \cdot \nabla \Pi_{E}^{\nabla} \phi_{j} \mathrm{~d} \boldsymbol{x}, \\
\left(\boldsymbol{K}_{\mathrm{S}}^{*}\right)_{i j} & :=\int_{E} \nabla\left(\mathrm{I}-\Pi_{E}^{\nabla}\right) \phi_{i} \cdot \nabla\left(\mathrm{I}-\Pi_{E}^{\nabla}\right) \phi_{j} \mathrm{~d} \boldsymbol{x} .
\end{aligned}
$$

Copyright (c) 2014 John Wiley \& Sons, Ltd.

Int. J. Numer. Meth. Engng 2014; 1:1-33

Prepared using nmeauth.cls 
- the replacement of the exact stability matrix $\boldsymbol{K}_{\mathrm{s}}^{*}$ with the inexact but computable matrix $\boldsymbol{K}_{\mathrm{s}}^{\mathrm{VEM}}$ yields the final virtual element decomposition of the stiffness matrix:

$$
\boldsymbol{K}^{\mathrm{VEM}}:=\boldsymbol{K}_{\mathrm{C}}^{\mathrm{VEM}}+\boldsymbol{K}_{\mathrm{S}}^{\mathrm{VEM}}
$$

with $\boldsymbol{K}_{\mathrm{s}}^{\mathrm{vEM}}$ defined by

$$
\left(\boldsymbol{K}_{\mathrm{S}}^{\mathrm{VEM}}\right)_{i j}:=\sum_{k, l=1}^{n_{E}}\left(\delta_{i k}-s_{k}^{(i)}\right) \boldsymbol{S}_{k l}\left(\delta_{j l}-s_{l}^{(j)}\right),
$$

where $\delta_{i k}$ is the Kronecker-delta symbol, $\boldsymbol{S}_{k l}$ are the elements of $\boldsymbol{S}$, a symmetric matrix whose construction is presented in Section 8.2, and $s_{k}^{(i)}$ are the coefficients of $\Pi_{E}^{\nabla} \phi_{i}$ in terms of the $\phi_{k}$ :

$$
\Pi_{E}^{\nabla} \phi_{i}=\sum_{k=1}^{n_{E}} s_{k}^{(i)} \phi_{k}
$$

\subsection{Definition of the projector $\Pi_{E}^{\nabla}$}

The element-wise projector $\Pi_{E}^{\nabla}$ clearly separates the consistency term from the stability term. It is defined as a Ritz-like projection operator that maps functions defined on $E$ to linear polynomials. Given a function $v$ defined on $E$, the projection $\Pi_{E}^{\nabla} v \in \mathcal{P}_{1}(E)$ of $v$ is defined by requiring that $v-\Pi_{E}^{\nabla} v$ is $a^{E}$-orthogonal to all linear polynomials:

$$
a^{E}\left(v-\Pi_{E}^{\nabla} v, p_{1}\right)=0 \quad \text { for all } p_{1} \in \mathcal{P}_{1}(E) .
$$

Because $a^{E}(\cdot, 1)$ is identically zero, (32) defines $\Pi_{E}^{\nabla} v$ only up to a constant. To complete the definition of $\Pi_{E}^{\nabla}$, we need another ingredient: a projection operator $\mathrm{P}_{0}$ onto the kernel of $a^{E}(\cdot, \cdot)$, that is, the space of constant functions, and we require that

$$
\mathrm{P}_{0}\left(v-\Pi_{E}^{\nabla} v\right)=0
$$

Copyright (C) 2014 John Wiley \& Sons, Ltd.

Int. J. Numer. Meth. Engng 2014; 1:1-33

Prepared using nmeauth.cls 
For the first-order VEM considered in this paper, the projection $\mathrm{P}_{0} v$ is defined as the meanvalue of $v$ over the $n_{E}$ vertices of $E[18]$ :

$$
\mathrm{P}_{0} v:=\bar{v}=\frac{1}{n_{E}} \sum_{i=1}^{n_{E}} v\left(\boldsymbol{x}_{i}\right) .
$$

On writing (32) explicitly, we obtain

$$
\int_{E} \nabla \Pi_{E}^{\nabla} v \cdot \nabla p_{1} \mathrm{~d} \boldsymbol{x}=\int_{E} \nabla v \cdot \nabla p_{1} \mathrm{~d} \boldsymbol{x}
$$

and because $\nabla \Pi_{E}^{\nabla} v$ and $\nabla p_{1}$ are constant vectors, (35) becomes

$$
\nabla \Pi_{E}^{\nabla} v \cdot \nabla p_{1}|E|=\nabla p_{1} \cdot \int_{E} \nabla v \mathrm{~d} \boldsymbol{x} .
$$

On choosing $p_{1}=x$ and $p_{1}=y$, we find that (36) is equivalent to

$$
\nabla \Pi_{E}^{\nabla} v=\frac{1}{|E|} \int_{E} \nabla v \mathrm{~d} \boldsymbol{x}
$$

Equation (37) determines $\Pi_{E}^{\nabla} v$ up to a constant:

$$
\Pi_{E}^{\nabla} v=\boldsymbol{x} \cdot \frac{1}{|E|} \int_{E} \nabla v \mathrm{~d} \boldsymbol{x}+C
$$

The constant $C$ is fixed by applying the projection $\mathrm{P}_{0}$ to both sides of (38), which yields

$$
\Pi_{E}^{\nabla} v=\left(\boldsymbol{x}-\mathrm{P}_{0} \boldsymbol{x}\right) \cdot \frac{1}{|E|} \int_{E} \nabla v \mathrm{~d} \boldsymbol{x}+\mathrm{P}_{0} v
$$

On noting that

$$
\mathrm{P}_{0} \boldsymbol{x}=\frac{1}{n_{E}} \sum_{i=1}^{n_{E}} \boldsymbol{x}_{i}=: \overline{\boldsymbol{x}}
$$

is the centroid of the set of nodes $\left\{\boldsymbol{x}_{1}, \ldots, \boldsymbol{x}_{n_{E}}\right\}$ and using the definition of $\mathrm{P}_{0} v$, we arrive at the following explicit formula for the $\Pi_{E}^{\nabla}$ projection:

$$
\Pi_{E}^{\nabla} v=(\boldsymbol{x}-\overline{\boldsymbol{x}}) \cdot \frac{1}{|E|} \int_{E} \nabla v \mathrm{~d} \boldsymbol{x}+\bar{v}
$$

Copyright (c) 2014 John Wiley \& Sons, Ltd.

Int. J. Numer. Meth. Engng 2014; 1:1-33

Prepared using nmeauth.cls 
Remark 1. The projection $\Pi_{E}^{\nabla} v$ is fully determined by knowing $v$ on $\partial E$ :

$$
\int_{E} \nabla v d \boldsymbol{x}=\int_{\partial E} v \boldsymbol{n} d s
$$

When $d=2$ and $v$ is piecewise linear on the edges of the polygon, the integral on the right-hand side of (41) is exactly computed through the trapezoidal rule. This requires only knowing the values of $v$ at the vertices of $E$.

\section{POLYGONAL AND POLYHEDRAL VIRTUAL ELEMENT METHOD}

In this section, we recall the first-order virtual element method for polygonal and polyhedral meshes. For a complete description of the theory and implementation of arbitrary-order VEM, we refer the interested reader to References [16, 18-20]. The connection with mimetic discretizations [21] is discussed in Reference [22].

\subsection{Definition of the space $V_{h}^{E}$ for $d=2$}

In two dimensions, a function $v_{h} \in V_{h}^{E}$ is characterized by the following properties:

- $v_{h}$ is continuous and piecewise linear on the polygonal boundary $\partial E$

- $\Delta v_{h}$ is a linear polynomial

- $\int_{E} v_{h} p_{1} \mathrm{~d} \boldsymbol{x}=\int_{E} \Pi_{E}^{\nabla} v_{h} p_{1} \mathrm{~d} \boldsymbol{x}$ for all linear polynomials $p_{1}$.

Note that the second condition is distinct from the one introduced in Reference [16] $\left(\Delta v_{h}=0\right)$. In addition, the third condition states that $\Pi_{E}^{\nabla} v_{h}$ is also the $L^{2}$-projection of $v_{h}$ onto linear polynomials [23]. These two modifications are adopted to enable the computation in 3D to be performed using only the values of $v_{h}$ at the vertices of $E$. The dimension of $V_{h}^{E}$ is equal to $n_{E}$, the number of vertices of $E$, and the values of $v_{h}$ at these vertices are taken as the degrees Copyright (C) 2014 John Wiley \& Sons, Ltd.

Int. J. Numer. Meth. Engng 2014; 1:1-33

Prepared using nmeauth.cls 
of freedom. Due to Remark 1, we are able to compute $\Pi_{E}^{\nabla} v_{h}$ exactly.

\subsection{Definition of the space $V_{h}^{E}$ for $d=3$}

In three dimensions, a function $v_{h} \in V_{h}^{E}$ is characterized by the following properties:

- the restriction of $v_{h}$ to a face $\mathbf{f}$ belongs to $V_{h}^{\mathrm{f}}$ as defined in (42)

- $\Delta v_{h}$ is a linear polynomial

- $\int_{E} v_{h} p_{1} \mathrm{~d} \boldsymbol{x}=\int_{E} \Pi_{E}^{\nabla} v_{h} p_{1} \mathrm{~d} \boldsymbol{x}$ for all linear polynomials $p_{1}$.

The dimension of $V_{h}^{E}$ is equal to $n_{E}$ and we take the values of $v_{h}$ at the vertices as degrees of freedom. The first property in (43) implies that $v_{h}$ is linear on each edge of $E$ and its trace on $f$ is uniquely determined by the values at the vertices of $f$. Consequently, $v_{h}$ is also continuous on $\partial E$. If $v_{h} \in V_{h}^{E}$, we can still compute $\Pi_{E}^{\nabla} v_{h}$ using only the vertex values of $v_{h}$. Indeed, a face $f$ of $E$ is a polygon; hence, we can define on $f$ the projector $\Pi_{f}^{\nabla}$ and the space $V_{h}^{f}$ as in (42). From (40), we only need to compute the mean value of $\nabla v_{h}$ and can write

$$
\int_{E} \nabla v_{h} \mathrm{~d} \boldsymbol{x}=\int_{\partial E} v_{h} \boldsymbol{n} \mathrm{d} s=\sum_{\mathbf{f} \subset \partial E} \boldsymbol{n}_{\mathbf{f}} \int_{\mathbf{f}} v_{h} \mathrm{~d} s=\sum_{\mathbf{f} \subset \partial E} \boldsymbol{n}_{\mathbf{f}} \int_{\mathbf{f}} \Pi_{\mathbf{f}}^{\nabla} v_{h} \mathrm{~d} s
$$

where the third relation in (42) with $p_{1}=1$ is used to obtain the last equality.

\subsection{Stiffness matrix of the VEM}

We assume that the degrees of freedom of a function $v_{h} \in V_{h}^{E}$ are the pointwise values of $v_{h}$ at the nodes $\boldsymbol{x}_{i}$ of the element $E$; that is,

$$
\operatorname{dof}_{i}\left(v_{h}\right)=v_{h}\left(\boldsymbol{x}_{i}\right)
$$

Remark 2. Let $\widehat{\operatorname{dof}}_{i}\left(\hat{v}_{h}\right)$ be the $i$-th degree of freedom of $\hat{v}_{h}$ defined on some fixed element and $\operatorname{dof}_{i}\left(v_{h}\right)$ the value of the corresponding degree of freedom on a rescaled element. If Copyright @ 2014 John Wiley \& Sons, Ltd.

Int. J. Numer. Meth. Engng 2014; 1:1-33

Prepared using nmeauth.cls 
$\operatorname{dof}_{i}\left(v_{h}\right)=\widehat{\operatorname{dof}}_{i}\left(\hat{v}_{h}\right)$ for all $i$, then the degrees of freedom are scale invariant. This property is trivially met if the degrees of freedom are pointwise values of $v_{h}$.

The Lagrange shape functions associated with the vertex degrees of freedom are given by $\left\{\phi_{1}, \ldots, \phi_{n_{E}}\right\}$, where $\phi_{i} \in V_{h}^{E}$ is identified by the following property:

$$
\phi_{i}\left(\boldsymbol{x}_{j}\right)=\delta_{i j}
$$

which leads to the following representation of functions $v_{h} \in V_{h}^{E}$ :

$$
v_{h}(\boldsymbol{x})=\sum_{i=1}^{n_{E}} v_{h}\left(\boldsymbol{x}_{i}\right) \phi_{i}(\boldsymbol{x})
$$

We now consider the decomposition $\boldsymbol{K}^{\mathrm{VEM}}=\boldsymbol{K}_{\mathrm{C}}^{\mathrm{VEM}}+\boldsymbol{K}_{\mathrm{s}}^{\mathrm{VEM}}$ given in (29) with the consistency matrix $\boldsymbol{K}_{\mathrm{C}}^{\mathrm{VEM}}$ and stability matrix $\boldsymbol{K}_{\mathrm{S}}^{\mathrm{VEM}}$ defined by (28b) and (30), respectively. On using (37), we rewrite the consistency term as follows:

$$
\begin{aligned}
\left(\boldsymbol{K}_{\mathrm{C}}^{\mathrm{VEM}}\right)_{i j} & =\int_{E} \nabla \Pi_{E}^{\nabla} \phi_{i} \cdot \nabla \Pi_{E}^{\nabla} \phi_{j} \mathrm{~d} \boldsymbol{x}=\int_{E}\left(\frac{1}{|E|} \int_{E} \nabla \phi_{i} \mathrm{~d} \boldsymbol{x}\right) \cdot\left(\frac{1}{|E|} \int_{E} \nabla \phi_{j} \mathrm{~d} \boldsymbol{x}\right) \mathrm{d} \boldsymbol{x} \\
& =|E|\left(\frac{1}{|E|} \int_{E} \nabla \phi_{i} \mathrm{~d} \boldsymbol{x}\right) \cdot\left(\frac{1}{|E|} \int_{E} \nabla \phi_{j} \mathrm{~d} \boldsymbol{x}\right)=\frac{1}{|E|} \int_{E} \nabla \phi_{i} \mathrm{~d} \boldsymbol{x} \cdot \int_{E} \nabla \phi_{j} \mathrm{~d} \boldsymbol{x} .
\end{aligned}
$$

The VEM stability term $\boldsymbol{K}_{\mathrm{s}}^{\mathrm{VEM}}$ replacing $\boldsymbol{K}_{\mathrm{s}}^{*}$ is constructed as follows:

1. Because $\Pi_{E}^{\nabla} \phi_{i}$ belongs to $\mathcal{P}_{1}(E)$, which is a subspace of $V_{h}^{E}, \Pi_{E}^{\nabla} \phi_{i}$ can in turn be written in terms of the $\phi_{k}$ 's:

$$
\Pi_{E}^{\nabla} \phi_{i}=\sum_{k=1}^{n_{E}} s_{k}^{(i)} \phi_{k}, \quad\left(\mathrm{I}-\Pi_{E}^{\nabla}\right) \phi_{i}=\sum_{k=1}^{n_{E}}\left(\delta_{i k}-s_{k}^{(i)}\right) \phi_{k} .
$$

From (45), we have

$$
\Pi_{E}^{\nabla} \phi_{i}=\sum_{k=1}^{n_{E}}\left(\Pi_{E}^{\nabla} \phi_{i}\right)\left(\boldsymbol{x}_{k}\right) \phi_{k} \quad \text { and } \quad \bar{\phi}_{i}=\frac{1}{n_{E}} \sum_{j=1}^{n_{E}} \phi_{i}\left(\boldsymbol{x}_{j}\right)=\frac{1}{n_{E}},
$$

and therefore $s_{k}^{(i)}$ defined in (47) is given by

$$
s_{k}^{(i)}=\operatorname{dof}_{k}\left(\Pi_{E}^{\nabla} \phi_{i}\right)=\left(\Pi_{E}^{\nabla} \phi_{i}\right)\left(\boldsymbol{x}_{k}\right)=\left(\boldsymbol{x}_{k}-\overline{\boldsymbol{x}}\right) \cdot \frac{1}{|E|} \int_{E} \nabla \phi_{i} \mathrm{~d} \boldsymbol{x}+\frac{1}{n_{E}} .
$$

Copyright (c) 2014 John Wiley \& Sons, Ltd.

Int. J. Numer. Meth. Engng 2014; 1:1-33

Prepared using nmeauth.cls 
2. Expanding $\left(\boldsymbol{K}_{\mathrm{s}}^{*}\right)_{i j}$ yields

$$
\begin{aligned}
\left(\boldsymbol{K}_{\mathrm{s}}^{*}\right)_{i j} & =\int_{E} \nabla\left(\mathrm{I}-\Pi_{E}^{\nabla}\right) \phi_{i} \cdot \nabla\left(\mathrm{I}-\Pi_{E}^{\nabla}\right) \phi_{j} \mathrm{~d} \boldsymbol{x} \\
& =\sum_{k, l=1}^{n_{E}}\left(\delta_{i k}-s_{k}^{(i)}\right)\left(\int_{E} \nabla \phi_{k} \cdot \nabla \phi_{l} \mathrm{~d} \boldsymbol{x}\right)\left(\delta_{j l}-s_{l}^{(j)}\right) .
\end{aligned}
$$

3. Instead of $\int_{E} \nabla \phi_{k} \cdot \nabla \phi_{l} \mathrm{~d} \boldsymbol{x}$, which is unknown, we pick a matrix $\boldsymbol{S}$ that lends stability: $\left(\boldsymbol{K}_{\mathrm{S}}^{\mathrm{VEM}}\right)_{i j}=\sum_{k, l=1}^{n_{E}}\left(\delta_{i k}-\left(\boldsymbol{x}_{k}-\overline{\boldsymbol{x}}\right) \cdot \frac{1}{|E|} \int_{E} \nabla \phi_{i} \mathrm{~d} \boldsymbol{x}-\frac{1}{n_{E}}\right) \boldsymbol{S}_{k l}\left(\delta_{j l}-\left(\boldsymbol{x}_{l}-\overline{\boldsymbol{x}}\right) \cdot \frac{1}{|E|} \int_{E} \nabla \phi_{j} \mathrm{~d} \boldsymbol{x}-\frac{1}{n_{E}}\right)$.

On using Remark 2, the degrees of freedom are scale-invariant, and the scaling properties of the stiffness matrix are determined only by $a^{E}\left(\phi_{k}, \phi_{l}\right)$, which scales like $s^{d-2}$ in $\mathbb{R}^{d}$ for any scaling factor $s$. In other words, using the notation of Remark 2, we have

$$
a^{E}\left(\phi_{k}, \phi_{l}\right)=s^{d-2} a^{\widehat{E}}\left(\widehat{\phi}_{k}, \widehat{\phi}_{l}\right)
$$

As shown in Reference [16], to ensure convergence we set $\boldsymbol{S}=h_{E}^{d-2} \boldsymbol{S}_{0}$, where $\boldsymbol{S}_{0}$ is any fixed symmetric and positive-definite matrix that does not depend on $h$. A simple choice is $\boldsymbol{S}_{0}=\boldsymbol{I}$.

Remark 3. On closer inspection of (48), it turns out that even a symmetric matrix that is positive-definite only on the kernel of $\Pi_{E}^{\nabla}$ can be used to define $\boldsymbol{S}$; see Reference [16] for details.

\section{BILINEAR QUADRILATERAL AND THE VEM}

In the section, we restrict $E$ to a convex quadrilateral $\Omega_{e}$, and choose $V_{h}^{E}$ as the space $\mathbb{Q}_{1}\left(\Omega_{e}\right)$ defined in (6). We show that

- the consistency matrix $\boldsymbol{K}_{\mathrm{C}}^{\mathrm{VEM}}$ coincides with $\boldsymbol{K}_{\mathrm{C}}^{\mathrm{FB}}$, see (13)

- the stabilization matrix $\boldsymbol{K}_{\mathrm{s}}^{\mathrm{VEM}}$ coincides with $\boldsymbol{K}_{\mathrm{s}}^{\mathrm{FB}}$ up to a constant factor, see (20).

Copyright (c) 2014 John Wiley \& Sons, Ltd.

Int. J. Numer. Meth. Engng 2014; 1:1-33

Prepared using nmeauth.cls 


\subsection{Consistency matrix}

The consistency matrix $\boldsymbol{K}_{\mathrm{C}}^{\mathrm{VEM}}$ is given by (46):

$$
\left(\boldsymbol{K}_{\mathrm{C}}^{\mathrm{VEM}}\right)_{i j}=\frac{1}{\left|\Omega_{e}\right|} \int_{\Omega_{e}} \nabla \phi_{i} \mathrm{~d} \boldsymbol{x} \cdot \int_{\Omega_{e}} \nabla \phi_{j} \mathrm{~d} \boldsymbol{x} .
$$

From the definitions in (12a) and (12b) for the vectors $\boldsymbol{b}_{\delta}$, it is observed that

$$
\left|\Omega_{e}\right|\left(\boldsymbol{b}_{1} \boldsymbol{b}_{1}^{\mathrm{T}}\right)_{i j}=\frac{1}{\left|\Omega_{e}\right|} \int_{\Omega_{e}} \frac{\partial \phi_{i}}{\partial x} \mathrm{~d} \boldsymbol{x} \int_{\Omega_{e}} \frac{\partial \phi_{j}}{\partial x} \mathrm{~d} \boldsymbol{x},
$$

so that

$$
\left(\boldsymbol{K}_{\mathrm{C}}^{\mathrm{FB}}\right)_{i j}=\left|\Omega_{e}\right| \sum_{\delta=1}^{2}\left(\boldsymbol{b}_{\delta} \boldsymbol{b}_{\delta}^{\mathrm{T}}\right)_{i j}=\frac{1}{\left|\Omega_{e}\right|} \int_{\Omega_{e}} \nabla \phi_{i} \mathrm{~d} \boldsymbol{x} \cdot \int_{\Omega_{e}} \nabla \phi_{j} \mathrm{~d} \boldsymbol{x}=\left(\boldsymbol{K}_{\mathrm{C}}^{\mathrm{VEM}}\right)_{i j}
$$

We first prove that the consistency matrix $\boldsymbol{K}_{\mathrm{C}}^{\mathrm{VEM}}$ given in (51) is identical to that obtained by applying one-point Gauss quadrature rule to the exact stiffness matrix $\int_{\Omega_{e}} \nabla \phi_{i} \cdot \nabla \phi_{j} \mathrm{~d} \boldsymbol{x}$. To this end, we begin by showing the equivalence stated in (15), namely that if $v_{h} \in \mathbb{Q}_{1}\left(\Omega_{e}\right)$, then approximating $\nabla v_{h}$ by its value at the vertex center $\overline{\boldsymbol{x}}$ of $\Omega_{e}$ is equivalent to taking its mean-value over $\Omega_{e}$, where $\overline{\boldsymbol{x}}$ is defined in (39). It suffices to show the equality for a particular basis of $\mathbb{Q}_{1}\left(\Omega_{e}\right)$; we choose the basis set $\{1, x, y, \Psi\}$ where $\Psi$ is the (unique) function in $\mathbb{Q}_{1}\left(\Omega_{e}\right)$ such that

$$
\Psi\left(\boldsymbol{x}_{1}\right)=-1 / 2, \quad \Psi\left(\boldsymbol{x}_{2}\right)=+1 / 2, \quad \Psi\left(\boldsymbol{x}_{3}\right)=-1 / 2, \quad \Psi\left(\boldsymbol{x}_{4}\right)=+1 / 2 .
$$

The function $\Psi$ on the reference biunit square is shown in Figure 3. It is noted that $\{1, x, y, \Psi\}$ is indeed a basis of $\mathbb{Q}_{1}\left(\Omega_{e}\right)$. For $v_{h}=1, x$, or $y$ the equality is evident because the gradient is constant; for $v_{h}=\Psi$, both terms are zero:

- the left-hand side of (15) is zero because the integral of $\Psi$ on each edge is zero; and

- the right-hand side of (15) is zero because $\Psi$ is zero at the midpoint of each edge, and hence it is identically zero along the two bimedians of $\Omega_{e}$ that meet precisely at $\overline{\boldsymbol{x}}$.

Copyright (C) 2014 John Wiley \& Sons, Ltd.

Int. J. Numer. Meth. Engng 2014; 1:1-33

Prepared using nmeauth.cls 


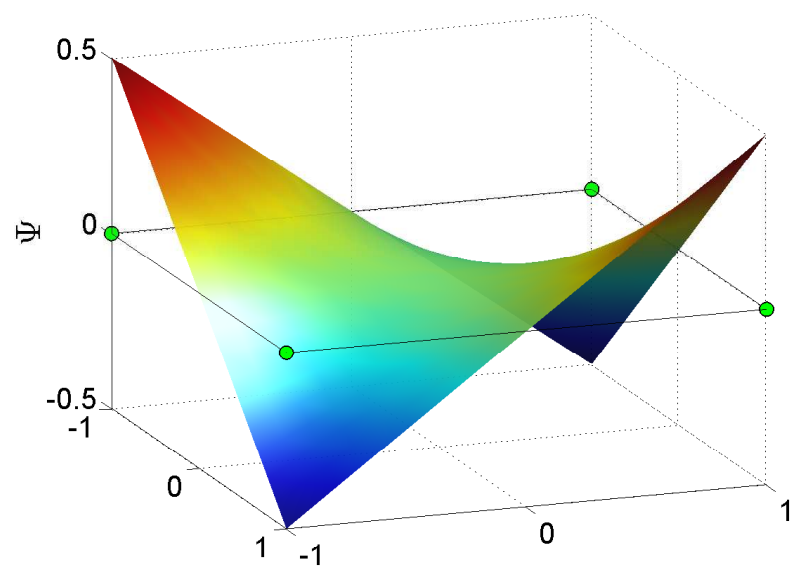

Figure 3. The function $\Psi(\boldsymbol{\xi})=\frac{\xi \eta}{2}$ on the reference biunit square.

Now, the consistency matrix $\boldsymbol{K}_{\mathrm{C}}^{\mathrm{VEM}}$ can be written as

$$
\left(\boldsymbol{K}_{\mathrm{C}}^{\mathrm{VEM}}\right)_{i j}=\frac{1}{\left|\Omega_{e}\right|} \int_{\Omega_{e}} \nabla \phi_{i} \mathrm{~d} \boldsymbol{x} \cdot \int_{\Omega_{e}} \nabla \phi_{j} \mathrm{~d} \boldsymbol{x}=\left|\Omega_{e}\right| \nabla \phi_{i}(\overline{\boldsymbol{x}}) \cdot \nabla \phi_{j}(\overline{\boldsymbol{x}}),
$$

which is equal to

$$
\left|\Omega_{e}\right| \boldsymbol{J}_{\boldsymbol{F}}^{-T}(0,0) \nabla_{\boldsymbol{\xi}} N_{i}(0,0) \cdot \boldsymbol{J}_{\boldsymbol{F}}^{-T}(0,0) \nabla_{\boldsymbol{\xi}} N_{j}(0,0),
$$

because $(0,0)$ is mapped to $\overline{\boldsymbol{x}}$ in the bilinear transformation $\boldsymbol{F}$ that maps the reference square $\Omega_{0}=(-1,1)^{2}$ to the convex quadrilateral $\Omega_{e}$.

We now establish that $\operatorname{det} \boldsymbol{J}_{\boldsymbol{F}}(0,0)=\left|\Omega_{e}\right| /\left|\Omega_{0}\right|$. Because $\operatorname{det} \boldsymbol{J}_{\boldsymbol{F}}(\xi, \eta)$ is bilinear in $\xi$ and $\eta$, the one-point Gauss quadrature rule on $\Omega_{0}$ integrates it exactly:

$$
\left|\Omega_{e}\right|=\int_{\Omega_{0}} \operatorname{det} \boldsymbol{J}_{\boldsymbol{F}}(\xi, \eta) \mathrm{d} \boldsymbol{\xi}=\left|\Omega_{0}\right| \operatorname{det} \boldsymbol{J}_{\boldsymbol{F}}(0,0) .
$$

On substituting expression (54) into (53), we obtain

$$
\left(\boldsymbol{K}_{\mathrm{C}}^{\mathrm{VEM}}\right)_{i j}=\left|\Omega_{0}\right| \boldsymbol{J}_{\boldsymbol{F}}^{-T}(0,0) \nabla_{\boldsymbol{\xi}} N_{i}(0,0) \cdot \boldsymbol{J}_{\boldsymbol{F}}^{-T}(0,0) \nabla_{\boldsymbol{\xi}} N_{j}(0,0) \operatorname{det} \boldsymbol{J}_{\boldsymbol{F}}(0,0),
$$


which is precisely the one-point Gauss quadrature formula applied to $\int_{\Omega_{e}} \nabla \phi_{i} \cdot \nabla \phi_{j} d \boldsymbol{x}$.

We can express $\boldsymbol{K}_{\mathrm{C}}^{\mathrm{VEM}}$ directly in terms of the vertex coordinates of the quadrilateral. On invoking the divergence theorem, we have

$$
\begin{aligned}
\int_{\Omega_{e}} \nabla \phi_{i} \mathrm{~d} \boldsymbol{x} & =\int_{\partial \Omega_{e}} \phi_{i} \boldsymbol{n} \mathrm{d} s=\sum_{k=1}^{4}\left(\int_{\mathbf{e}_{k}} \phi_{i} \mathrm{~d} s\right) \boldsymbol{n}_{k} \\
& =\left(\int_{\mathbf{e}_{i-1}} \phi_{i} \mathrm{~d} s\right) \boldsymbol{n}_{i-1}+\left(\int_{\mathbf{e}_{i}} \phi_{i} \mathrm{~d} s\right) \boldsymbol{n}_{i}=\frac{\left|\mathbf{e}_{i-1}\right|}{2} \boldsymbol{n}_{i-1}+\frac{\left|\mathbf{e}_{i}\right|}{2} \boldsymbol{n}_{i}
\end{aligned}
$$

because $\phi_{i}$ is an affine function on each edge $\mathrm{e}_{k}$ of the quadrilateral $\Omega_{e}$. Letting $\boldsymbol{d}_{i}$ be the vector joining vertex $\boldsymbol{x}_{i-1}$ to $\boldsymbol{x}_{i+1}$, we have

$$
\frac{\left|\mathrm{e}_{i-1}\right|}{2} \boldsymbol{n}_{i-1}+\frac{\left|\mathrm{e}_{i}\right|}{2} \boldsymbol{n}_{i}=\frac{1}{2} \boldsymbol{d}_{i}^{\perp}
$$

where $\perp$ refers to a clockwise rotation of $90^{\circ}$. Hence,

$$
\left(\boldsymbol{K}_{\mathrm{C}}^{\mathrm{VEM}}\right)_{i j}:=\frac{1}{4\left|\Omega_{e}\right|} \boldsymbol{d}_{i}^{\perp} \cdot \boldsymbol{d}_{j}^{\perp}=\frac{1}{4\left|\Omega_{e}\right|} \boldsymbol{d}_{i} \cdot \boldsymbol{d}_{j} .
$$

For a convex quadrilateral, we have

$$
\boldsymbol{d}_{3}=-\boldsymbol{d}_{1}, \quad \boldsymbol{d}_{4}=-\boldsymbol{d}_{2}
$$

and therefore the matrix $\boldsymbol{K}_{\mathrm{C}}^{\mathrm{VEM}}$ has the block structure

$$
\boldsymbol{K}_{\mathrm{C}}^{\mathrm{VEM}}=\left[\begin{array}{cc}
+\boldsymbol{U} & -\boldsymbol{U} \\
-\boldsymbol{U} & +\boldsymbol{U}
\end{array}\right], \quad \text { where } \quad \boldsymbol{U}=\frac{1}{4\left|\Omega_{e}\right|}\left[\begin{array}{cc}
\boldsymbol{d}_{1} \cdot \boldsymbol{d}_{1} & \boldsymbol{d}_{1} \cdot \boldsymbol{d}_{2} \\
\boldsymbol{d}_{1} \cdot \boldsymbol{d}_{2} & \boldsymbol{d}_{2} \cdot \boldsymbol{d}_{2}
\end{array}\right] .
$$

\subsection{Stabilization matrix}

Instead of starting from (49), we compute the stabilization matrix $\boldsymbol{K}_{\mathrm{S}}^{\mathrm{VEM}}$ as follows. Because there is only one nonlinear function in $\mathbb{Q}_{1}\left(\Omega_{e}\right)$, we can explicitly compute the expansion of $\left(\mathrm{I}-\Pi_{E}^{\nabla}\right) \phi_{i}$ in terms of the basis functions $\phi_{k}$; that is, we directly compute the coefficients $\left(\delta_{i k}-s_{k}^{(i)}\right)$ of $(47)$.

Copyright (c) 2014 John Wiley \& Sons, Ltd.

Int. J. Numer. Meth. Engng 2014; 1:1-33

Prepared using nmeauth.cls 
We start by finding a function in the kernel of the $\Pi_{E}^{\nabla}$ operator, that is, a zero-energy mode. A candidate is the function $\Psi$ in (52), which corresponds to the vector $\boldsymbol{\Gamma}$ in (16). From the definition of $\Psi$, we have

$$
\Psi=\frac{1}{2}\left(-\phi_{1}+\phi_{2}-\phi_{3}+\phi_{4}\right)=\frac{1}{2} \sum_{k=1}^{4}(-1)^{k} \phi_{k} .
$$

Let us show that the function $\Psi$ is a zero-energy mode; that is, $\Pi_{E}^{\nabla} \Psi=0$. From (40), we have

$$
\Pi_{E}^{\nabla} \Psi=(\boldsymbol{x}-\overline{\boldsymbol{x}}) \cdot \frac{1}{\left|\Omega_{e}\right|} \int_{\Omega_{e}} \nabla \Psi \mathrm{d} \boldsymbol{x}+\bar{\Psi}
$$

From the divergence theorem, it follows that

$$
\int_{\Omega_{e}} \nabla \Psi \mathrm{d} \boldsymbol{x}=\int_{\partial \Omega_{e}} \Psi \boldsymbol{n} \mathrm{d} s=\sum_{k=1}^{4}\left(\int_{e_{k}} \Psi \mathrm{d} s\right) \boldsymbol{n}_{k}=\mathbf{0}
$$

because $\Psi$ has zero mean-value on each edge. Since $\bar{\Psi}=0$, it follows that $\Pi_{E}^{\nabla} \Psi=0$. Furthermore, (45) implies that

$$
1=\sum_{i=1}^{4} \phi_{i}, \quad x=\sum_{i=1}^{4} x_{i} \phi_{i} \quad \text { and } \quad y=\sum_{i=1}^{4} y_{i} \phi_{i} .
$$

Therefore, the linear transformation from the basis $\left\{\phi_{1}, \phi_{2}, \phi_{3}, \phi_{4}\right\}$ to the basis $\{1, x, y, \Psi\}$ of $\mathbb{Q}_{1}\left(\Omega_{e}\right)$ is:

$$
\left[\begin{array}{cccc}
1 & 1 & 1 & 1 \\
x_{1} & x_{2} & x_{3} & x_{4} \\
y_{1} & y_{2} & y_{3} & y_{4} \\
-1 / 2 & +1 / 2 & -1 / 2 & +1 / 2
\end{array}\right]\left[\begin{array}{l}
\phi_{1} \\
\phi_{2} \\
\phi_{3} \\
\phi_{4}
\end{array}\right]=\left[\begin{array}{c}
1 \\
x \\
y \\
\Psi
\end{array}\right] .
$$

Let us denote by $T_{i}$ the signed area of the triangle obtained by removing the vertex $\boldsymbol{x}_{i}$ from Copyright (C) 2014 John Wiley \& Sons, Ltd. Int. J. Numer. Meth. Engng 2014; 1:1-33 Prepared using nmeauth.cls 
the quadrilateral $\Omega_{e}$. Therefore,

$$
\begin{aligned}
& T_{1}:=\frac{1}{2} \operatorname{det}\left[\begin{array}{ccc}
1 & 1 & 1 \\
x_{2} & x_{3} & x_{4} \\
y_{2} & y_{3} & y_{4}
\end{array}\right], T_{2}:=\frac{1}{2} \operatorname{det}\left[\begin{array}{ccc}
1 & 1 & 1 \\
x_{1} & x_{3} & x_{4} \\
y_{1} & y_{3} & y_{4}
\end{array}\right], \\
& T_{3}:=\frac{1}{2} \operatorname{det}\left[\begin{array}{lll}
1 & 1 & 1 \\
x_{1} & x_{2} & x_{4} \\
y_{1} & y_{2} & y_{4}
\end{array}\right], T_{4}:=\frac{1}{2} \operatorname{det}\left[\begin{array}{lll}
1 & 1 & 1 \\
x_{1} & x_{2} & x_{3} \\
y_{1} & y_{2} & y_{3}
\end{array}\right] .
\end{aligned}
$$

Now, let us consider the coefficient matrix of the linear system in (56). Carrying out the expansion with respect to the last row, it is verified that its determinant is equal to $2\left|\Omega_{e}\right|$. By directly solving system (56) through the Cramer's rule, we see that

$$
\phi_{1}=\frac{\left[\begin{array}{cccc}
1 & 1 & 1 & 1 \\
x & x_{2} & x_{3} & x_{4} \\
y & y_{2} & y_{3} & y_{4} \\
\Psi & +1 / 2 & -1 / 2 & +1 / 2
\end{array}\right]}{2\left|\Omega_{e}\right|}=\left(a_{1}+b_{1} x+c_{1} y\right)-\frac{2 T_{1}}{2\left|\Omega_{e}\right|} \Psi
$$

and in general one can write

$$
\phi_{i}=\left(a_{i}+b_{i} x+c_{i} y\right)+(-1)^{i} \frac{T_{i}}{\left|\Omega_{e}\right|} \Psi, \quad i=1, \ldots, 4,
$$

where the coefficients $a_{i}, b_{i}, c_{i}$ depend on the coordinates of the vertices. Since $\Pi_{E}^{\nabla} \Psi=0$, we have $\Pi_{E}^{\nabla} \phi_{i}=a_{i}+b_{i} x+c_{i} y$, so that

$$
\left(\mathrm{I}-\Pi_{E}^{\nabla}\right) \phi_{i}=(-1)^{i} \frac{T_{i}}{\left|\Omega_{e}\right|} \Psi=(-1)^{i} \frac{T_{i}}{\left|\Omega_{e}\right|}\left(\frac{1}{2} \sum_{k=1}^{4}(-1)^{k} \phi_{k}\right),
$$

that is,

$$
\delta_{i k}-s_{k}^{(i)}=(-1)^{i+k} \frac{T_{i}}{2\left|\Omega_{e}\right|} .
$$

Copyright (C) 2014 John Wiley \& Sons, Ltd.

Int. J. Numer. Meth. Engng 2014; 1:1-33

Prepared using nmeauth.cls 
Hence, the stabilization matrix $\boldsymbol{K}_{\mathrm{S}}^{\mathrm{VEM}}$ is written as

$$
\begin{aligned}
\left(\boldsymbol{K}_{\mathrm{s}}^{\mathrm{VEM}}\right)_{i j} & =\sum_{k, l=1}^{4}\left(\delta_{i k}-s_{k}^{(i)}\right) \boldsymbol{S}_{k l}\left(\delta_{j l}-s_{l}^{(j)}\right) \\
& =\sum_{k, l=1}^{4}\left((-1)^{i+k} \frac{T_{i}}{2\left|\Omega_{e}\right|}\right) \boldsymbol{S}_{k l}\left((-1)^{j+l} \frac{T_{j}}{2\left|\Omega_{e}\right|}\right) \\
& =(-1)^{i+j} \frac{T_{i} T_{j}}{\left|\Omega_{e}\right|^{2}}\left(\frac{1}{4} \sum_{k, l=1}^{4}(-1)^{k+l} \boldsymbol{S}_{k l}\right)
\end{aligned}
$$

Since $d=2$, we let $\boldsymbol{S}=\boldsymbol{I}$ in (57) and obtain

$$
\left(\boldsymbol{K}_{\mathrm{S}}^{\mathrm{VEM}}\right)_{i j}=(-1)^{i+j} \frac{T_{i} T_{j}}{\left|\Omega_{e}\right|^{2}}
$$

On defining the nondimensional quantities $T_{i}^{\prime}=T_{i} /\left|\Omega_{e}\right|$, and setting

$$
\gamma:=\left[\begin{array}{llll}
+T_{1}^{\prime} & -T_{2}^{\prime} & +T_{3}^{\prime} & -T_{4}^{\prime}
\end{array}\right]^{\mathrm{T}}
$$

the matrix in (58) is written as

$$
\boldsymbol{K}_{\mathrm{S}}^{\mathrm{VEM}}=\gamma \gamma^{\mathrm{T}}
$$

The Flanagan-Belytschko vector $\boldsymbol{\gamma}$ in (19) is equal to the VEM vector $\boldsymbol{\gamma}$ defined in (59) divided by 2 ; hence

$$
\boldsymbol{K}_{\mathrm{s}}^{\mathrm{FB}}=\frac{\left|\Omega_{e}\right| \bar{\epsilon}}{4} \boldsymbol{K}_{\mathrm{s}}^{\mathrm{vEM}} .
$$

If $\tau$ is a positive parameter independent of the mesh, the stiffness matrix of the VEM for $\boldsymbol{S}=\tau \boldsymbol{I}$ is given by $[16]$

$$
\boldsymbol{K}^{\mathrm{VEM}}=\boldsymbol{K}_{\mathrm{C}}^{\mathrm{VEM}}+\tau \boldsymbol{\gamma} \boldsymbol{\gamma}^{\mathrm{T}}
$$

which always results in a scheme that is convergent. Hence, in order to yield a convergent scheme, the parameter $\bar{\epsilon}$ that appears in the Flanagan-Belytschko stabilization matrix must scale like $h_{e}^{-2}$.

Copyright (C) 2014 John Wiley \& Sons, Ltd.

Int. J. Numer. Meth. Engng 2014; 1:1-33

Prepared using nmeauth.cls 
Remark 4. When $\Omega_{e}$ is a parallelogram, we have

$$
\gamma=\frac{1}{2}\left[\begin{array}{llll}
+1 & -1 & +1 & -1
\end{array}\right]^{\mathrm{T}}
$$

and

$$
\gamma \gamma^{\mathrm{T}}=\frac{1}{4}\left[\begin{array}{cccc}
+1 & -1 & +1 & -1 \\
-1 & +1 & -1 & +1 \\
+1 & -1 & +1 & -1 \\
-1 & +1 & -1 & +1
\end{array}\right],
$$

which when scaled by a factor of 2 coincides with the hourglass control matrix of Hansbo [24].

Remark 5. Taking $\boldsymbol{S}=\tau \boldsymbol{I}$ corresponds to approximating the energy of function $\Psi$ with $\tau$ :

$$
\int_{\Omega_{e}}|\nabla \Psi|^{2} d \boldsymbol{x}=\sum_{k, l=1}^{n_{E}} \frac{(-1)^{k+l}}{4} \int_{\Omega_{e}} \nabla \phi_{k} \cdot \nabla \phi_{l} d \boldsymbol{x} \approx \sum_{k, l=1}^{n_{E}} \frac{(-1)^{k+l}}{4} \tau \delta_{k l}=\tau .
$$

In general, we have

$$
\int_{\Omega_{e}}|\nabla \Psi|^{2} d \boldsymbol{x}=\int_{\Omega_{0}}\left|\boldsymbol{J}_{\boldsymbol{F}}^{-T} \nabla_{\boldsymbol{\xi}} \Psi\right|^{2} \operatorname{det}\left(\boldsymbol{J}_{\boldsymbol{F}}\right) d \boldsymbol{\xi}
$$

which usually can not be integrated in closed-form. The following identity holds for the exact stiffness matrix $\boldsymbol{K}$ for any quadrilateral $\Omega_{e}$ :

$$
\boldsymbol{K}=\boldsymbol{K}_{\mathrm{C}}^{\mathrm{VEM}}+\left[\int_{\Omega_{e}}|\nabla \Psi|^{2} d \boldsymbol{x}\right] \boldsymbol{\gamma} \boldsymbol{\gamma}^{\mathrm{T}},
$$

where $\boldsymbol{K}_{\mathrm{C}}^{\mathrm{VEM}}$ and $\boldsymbol{\gamma}$ are defined in (51) and (59), respectively. Because

$$
\Psi=\frac{1}{2}\left(-\phi_{1}+\phi_{2}-\phi_{3}+\phi_{4}\right) \quad \text { and } \quad \phi_{1}+\phi_{2}+\phi_{3}+\phi_{4}=1,
$$

we have

$$
\Psi=\frac{1}{2}-\phi_{1}-\phi_{3}=\frac{1}{2}+\phi_{2}+\phi_{4}
$$

so that

$$
\int_{\Omega_{e}}|\nabla \Psi|^{2} d \boldsymbol{x}=\int_{\Omega_{e}}\left|\nabla\left(\phi_{1}+\phi_{3}\right)\right|^{2} d \boldsymbol{x}=\int_{\Omega_{e}}\left|\nabla\left(\phi_{2}+\phi_{4}\right)\right|^{2} d \boldsymbol{x} .
$$

Copyright (c) 2014 John Wiley \& Sons, Ltd.

Int. J. Numer. Meth. Engng 2014; 1:1-33

Prepared using nmeauth.cls 
In Appendix B.1, on using $\tau$ as a parameter, the stencils that are obtained from the VEM for a uniform square grid are compared to well-known finite-difference stencils, whereas in Appendix B.2 we exploit the VEM decomposition (63) for a few special quadrilaterals.

\section{TRILINEAR HEXAHEDRON AND THE VEM}

We now consider the case when $E$ is a hexahedron $\Omega_{e}$ and $V_{h}^{E}$ is the space $\mathbb{Q}_{1}\left(\Omega_{e}\right)$ defined in (8). We show that

- the consistency matrix $\boldsymbol{K}_{\mathrm{C}}^{\mathrm{VEM}}$ coincides with that obtained via reduced integration (mean-gradient formulation) of the exact bilinear form.

- The stabilization matrix $\boldsymbol{K}_{\mathrm{S}}^{\mathrm{VEM}}$ coincides with the Flanagan-Belytschko stabilization of the hourglass modes for a particular choice of the matrix $\boldsymbol{S}$.

\subsection{Consistency matrix}

The consistency matrix $\boldsymbol{K}_{\mathrm{C}}^{\mathrm{VEM}}$ is given by (46):

$$
\left(\boldsymbol{K}_{\mathrm{C}}^{\mathrm{VEM}}\right)_{i j}=\frac{1}{\left|\Omega_{e}\right|} \int_{\Omega_{e}} \nabla \phi_{i} \mathrm{~d} \boldsymbol{x} \cdot \int_{\Omega_{e}} \nabla \phi_{j} \mathrm{~d} \boldsymbol{x} .
$$

Following the same arguments as in Section 8, we conclude that

$$
\boldsymbol{K}_{\mathrm{C}}^{\mathrm{VEM}}=\boldsymbol{K}_{\mathrm{C}}^{\mathrm{FB}},
$$

that is, the VEM consistency matrix (64) and the Flanagan-Belytschko consistency matrix (13) coincide.

As already noted, for the eight-node hexahedral isoparametric element, use of the one-point Gauss quadrature to integrate $\nabla \phi_{i}$ is not equivalent to taking the mean-value of $\nabla \phi_{i}$ over the element. In other words, (15) does not hold in three dimensions. The integral of the gradient

Copyright (C) 2014 John Wiley \& Sons, Ltd.

Int. J. Numer. Meth. Engng 2014; 1:1-33

Prepared using nmeauth.cls 
of the basis functions can be explicitly computed by mapping back to the reference element $\Omega_{0}[8]:$

$$
\int_{\Omega_{e}} \nabla \phi_{i} \mathrm{~d} \boldsymbol{x}=\int_{\Omega_{0}} \boldsymbol{J}_{\boldsymbol{F}}^{-\mathrm{T}} \nabla_{\boldsymbol{\xi}} N_{i} \operatorname{det}\left(\boldsymbol{J}_{\boldsymbol{F}}\right) \mathrm{d} \boldsymbol{\xi}
$$

Equation (65) — when combined with (46) and (49) — permits the virtual element decomposition to be applicable to hexahedral elements with curved faces that are the image in the physical space of the reference biunit cube.

\subsection{Stabilization matrix}

Using (49), we take $\boldsymbol{S}=h_{e} \boldsymbol{I}$ and obtain a convergent virtual element method, which is confirmed by our numerical experiments in Section 10. To draw comparisons between the stabilization matrices $\boldsymbol{K}_{\mathrm{S}}^{\mathrm{VEM}}$ and $\boldsymbol{K}_{\mathrm{S}}^{\mathrm{FB}}$, we need to discuss the conditions on the matrix $\boldsymbol{S}$. As pointed out in Remark 3, matrix $\boldsymbol{S}$ need not be symmetric and strictly positive-definite; it can just be positive-definite on the linear subspace of vectors representing the kernel of $\Pi_{E}^{\nabla}$, which corresponds to the nonpolynomial functions. Hence, the kernel of $\boldsymbol{S}$ can include the vectors representing polynomial functions, and in particular, all constant functions, which are polynomials of degree zero. If so, then (49) simplifies to

$$
\left(\boldsymbol{K}_{\mathrm{S}}^{\mathrm{VEM}}\right)_{i j}=\sum_{k, l=1}^{8}\left(\delta_{i k}-\boldsymbol{x}_{k} \cdot \frac{1}{|E|} \int_{E} \nabla \phi_{i} \mathrm{~d} \boldsymbol{x}\right) \boldsymbol{S}_{k l}\left(\delta_{j l}-\boldsymbol{x}_{l} \cdot \frac{1}{|E|} \int_{E} \nabla \phi_{j} \mathrm{~d} \boldsymbol{x}\right),
$$

where we have left out the terms that do not depend on the indices $k$ and $l$; because the constant lies in the kernel of $\boldsymbol{S}$, the omitted terms do not contribute to $\boldsymbol{K}_{\mathrm{s}}^{\mathrm{VEM}}$.

Now, we are ready to show that the Flanagan-Belytschko stabilization matrix $\boldsymbol{K}_{\mathrm{s}}^{\mathrm{FB}}$ is equal

Copyright (C) 2014 John Wiley \& Sons, Ltd.

Int. J. Numer. Meth. Engng 2014; 1:1-33

Prepared using nmeauth.cls 
to the stabilization matrix $\boldsymbol{K}_{\mathrm{s}}^{\mathrm{VEM}}$ with $\boldsymbol{S}$ given by

$$
\boldsymbol{S}=\left|\Omega_{e}\right| \bar{\epsilon} \sum_{\alpha=1}^{4} \Gamma_{\alpha} \Gamma_{\alpha}^{\mathrm{T}}=\left|\Omega_{e}\right| \bar{\epsilon}\left[\begin{array}{rrrrrrrrr}
+4 & -2 & 0 & -2 & -2 & 0 & +2 & 0 \\
-2 & +4 & -2 & 0 & 0 & -2 & 0 & +2 \\
0 & -2 & +4 & -2 & +2 & 0 & -2 & 0 \\
-2 & 0 & -2 & +4 & 0 & +2 & 0 & -2 \\
-2 & 0 & +2 & 0 & +4 & -2 & 0 & -2 \\
0 & -2 & 0 & +2 & -2 & +4 & -2 & 0 \\
+2 & 0 & -2 & 0 & 0 & -2 & +4 & -2 \\
0 & +2 & 0 & -2 & -2 & 0 & -2 & +4
\end{array}\right] \text {, }
$$

where we recall that the vectors $\boldsymbol{\Gamma}_{\alpha}$ are defined in (22).

We first observe that $\boldsymbol{S}$ has the constant vector in the kernel because each $\boldsymbol{\Gamma}_{\alpha}$ has the constant vector in the kernel. Then, the VEM stabilization matrix (66) with $\boldsymbol{S}$ given by (67) takes the form:

$$
\left(\boldsymbol{K}_{\mathrm{S}}^{\mathrm{VEM}}\right)_{i j}=\sum_{k, l=1}^{8}\left[\delta_{i k}-\boldsymbol{x}_{k} \cdot \frac{1}{\left|\Omega_{e}\right|} \int_{\Omega_{e}} \nabla \phi_{i} d \boldsymbol{x}\right]\left[\left|\Omega_{e}\right| \bar{\epsilon} \sum_{\alpha=1}^{4} \boldsymbol{\Gamma}_{\alpha k} \boldsymbol{\Gamma}_{\alpha l}\right]\left[\delta_{j l}-\boldsymbol{x}_{l} \cdot \frac{1}{\left|\Omega_{e}\right|} \int_{\Omega_{e}} \nabla \phi_{j} d \boldsymbol{x}\right]
$$

or

$$
\left(\boldsymbol{K}_{\mathrm{s}}^{\mathrm{VEM}}\right)_{i j}=\left|\Omega_{e}\right| \bar{\epsilon} \sum_{\alpha=1}^{4}\left\{\sum_{k=1}^{8}\left[\delta_{i k}-\boldsymbol{x}_{k} \cdot \frac{1}{\left|\Omega_{e}\right|} \int_{\Omega_{e}} \nabla \phi_{i} d \boldsymbol{x}\right] \boldsymbol{\Gamma}_{\alpha k}\right\}\left\{\sum_{l=1}^{8}\left[\delta_{j l}-\boldsymbol{x}_{l} \cdot \frac{1}{\left|\Omega_{e}\right|} \int_{\Omega_{e}} \nabla \phi_{j} d \boldsymbol{x}\right] \boldsymbol{\Gamma}_{\alpha l}\right\},
$$

and therefore

$$
\left(\boldsymbol{K}_{\mathrm{S}}^{\mathrm{VEM}}\right)_{i j}=\left|\Omega_{e}\right| \bar{\epsilon} \sum_{\alpha=1}^{4}\left\{\boldsymbol{\Gamma}_{\alpha i}-\sum_{k=1}^{8}\left(\boldsymbol{x}_{k} \cdot \frac{1}{\left|\Omega_{e}\right|} \int_{\Omega_{e}} \nabla \phi_{i}\right) \boldsymbol{\Gamma}_{\alpha k}\right\}\left\{\boldsymbol{\Gamma}_{\alpha j}-\sum_{l=1}^{8}\left(\boldsymbol{x}_{l} \cdot \frac{1}{\left|\Omega_{e}\right|} \int_{\Omega_{e}} \nabla \phi_{j}\right) \boldsymbol{\Gamma}_{\alpha l}\right\}
$$

On introducing the symbol

$$
\gamma_{\alpha i}:=\boldsymbol{\Gamma}_{\alpha i}-\sum_{k=1}^{8}\left(\boldsymbol{x}_{k} \cdot \frac{1}{\left|\Omega_{e}\right|} \int_{\Omega_{e}} \nabla \phi_{i} d \boldsymbol{x}\right) \boldsymbol{\Gamma}_{\alpha k}
$$

the VEM stabilization term can be rewritten as

$$
\boldsymbol{K}_{\mathrm{S}}^{\mathrm{VEM}}=\left|\Omega_{e}\right| \bar{\epsilon} \sum_{\alpha=1}^{4} \gamma_{\alpha} \gamma_{\alpha}^{\mathrm{T}}
$$

Copyright (C) 2014 John Wiley \& Sons, Ltd.

Int. J. Numer. Meth. Engng 2014; 1:1-33

Prepared using nmeauth.cls 
Comparing the previous equality to (25), we only have to check that $\gamma$ defined in (24) is equal to $\gamma$ defined in (68). Because

$$
\left[\left(\boldsymbol{\Gamma}_{\alpha}^{\mathrm{T}} \boldsymbol{X}\right) \boldsymbol{b}_{1}\right]_{i}=\left(\sum_{k=1}^{8} \boldsymbol{\Gamma}_{\alpha k} x_{k}\right) \frac{1}{\left|\Omega_{e}\right|} \int_{\Omega_{e}} \frac{\partial \phi_{i}}{\partial x} \mathrm{~d} \boldsymbol{x}=\sum_{k=1}^{8}\left(x_{k} \frac{1}{\left|\Omega_{e}\right|} \int_{\Omega_{e}} \frac{\partial \phi_{i}}{\partial x} \mathrm{~d} \boldsymbol{x}\right) \boldsymbol{\Gamma}_{\alpha k},
$$

we have

$$
\left[\boldsymbol{\Gamma}_{\alpha}-\left(\boldsymbol{\Gamma}_{\alpha}^{\mathrm{T}} \boldsymbol{X}\right) \boldsymbol{b}_{1}-\left(\boldsymbol{\Gamma}_{\alpha}^{\mathrm{T}} \boldsymbol{Y}\right) \boldsymbol{b}_{2}-\left(\boldsymbol{\Gamma}_{\alpha}^{\mathrm{T}} \boldsymbol{Z}\right) \boldsymbol{b}_{3}\right]_{i}=\boldsymbol{\Gamma}_{\alpha i}-\sum_{k=1}^{8}\left(\boldsymbol{x}_{k} \cdot \frac{1}{\left|\Omega_{e}\right|} \int_{\Omega_{e}} \nabla \phi_{i}\right) \boldsymbol{\Gamma}_{\alpha k},
$$

so that (24) and (68) coincide. We conclude that $\boldsymbol{K}_{\mathrm{S}}^{\mathrm{FB}}=\boldsymbol{K}_{\mathrm{S}}^{\mathrm{VEM}}$ for $\boldsymbol{S}$ given by (67) and that optimal convergence is ensured if the parameter $\bar{\epsilon}$ of (69) scales like $h_{e}^{-2}$ [16]. This fact is confirmed by the numerical experiments presented in Section 10. Furthermore, we need to check that matrix $S$ is positive-definite on the linear subspace of $V_{h}^{E}$ that represents the kernel of $\Pi_{E}^{\nabla}$, that is, the nonlinear functions. To this end, it suffices to show that in the kernel of $\boldsymbol{S}$ there are no vectors of $V_{h}^{E}$ corresponding to the nonlinear functions, and this is precisely what has been shown in Reference [25].

\section{NUMERICAL EXAMPLES}

\subsection{Two-dimensional problems}

We consider the domain $\Omega=(0,1)^{2}$, which is partitioned into a union of nonoverlapping elements. We first consider convex quadrilateral meshes and compare the solutions obtained by VEM and bilinear FEM on these meshes, and then present the virtual element solutions on more general polygonal meshes.

10.1.1. Convex quadrilateral meshes. A sequence of five unstructured convex quadrilateral meshes are used to discretize the domain. In Table I, the various mesh parameters for the five 


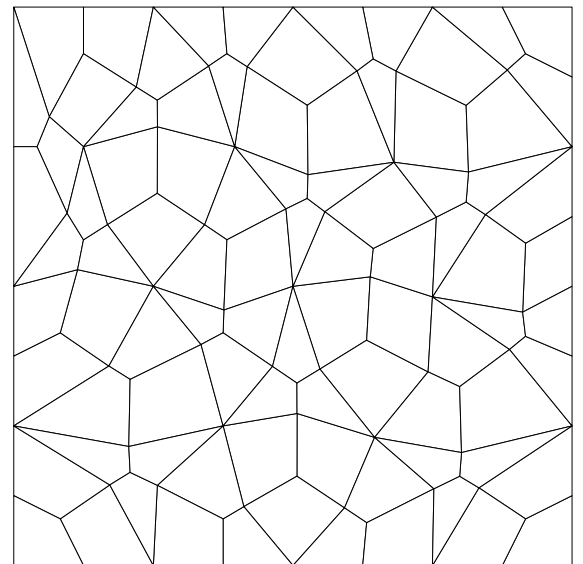

(a)

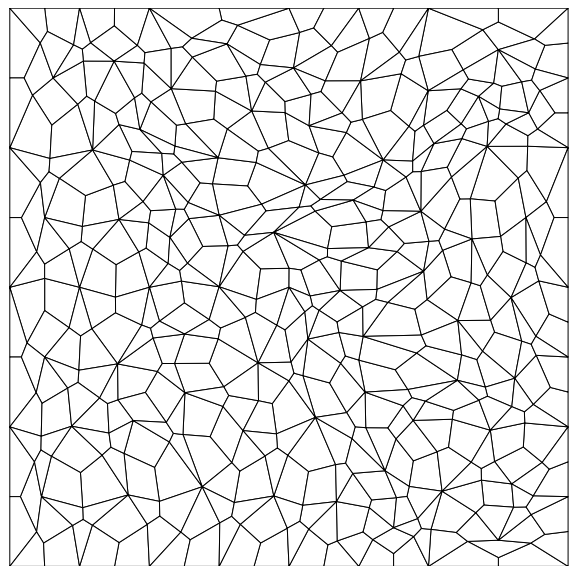

(b)

Figure 4. (a) First and (b) second convex quadrilateral meshes of the sequence.

meshes are presented ( $h$ is the average diameter of an element). The first and second meshes in the sequence are shown in Figure 4.

\begin{tabular}{|c|c|c|c|}
\hline Mesh & $\begin{array}{c}\text { Number of } \\
\text { elements }\end{array}$ & $\begin{array}{c}\text { Number of } \\
\text { nodes }\end{array}$ & $\begin{array}{c}\text { Element } \\
\text { diameter } h\end{array}$ \\
\hline$a$ & 87 & 103 & $1.8 \times 10^{-1}$ \\
$b$ & 450 & 475 & $8.2 \times 10^{-2}$ \\
$c$ & 957 & 991 & $5.6 \times 10^{-2}$ \\
$d$ & 4635 & 4707 & $2.5 \times 10^{-2}$ \\
$e$ & 9402 & 9521 & $1.8 \times 10^{-2}$ \\
\hline
\end{tabular}

Table I. Mesh parameters for the unstructured quadrilateral meshes.

We first confirm that the virtual element scheme presented in Section 8 passes the patch Copyright (C) 2014 John Wiley \& Sons, Ltd. Int. J. Numer. Meth. Engng 2014; 1:1-33

Prepared using nmeauth.cls 
test. To this end, we let $f(\boldsymbol{x})=0$ and $g(\boldsymbol{x})=3 x-2 y+1$ in (1), so that the exact solution is $u(\boldsymbol{x})=g(\boldsymbol{x})$. The relative $L^{2}$-errors of the VEM listed in Table II clearly show that the patch test is passed.

\begin{tabular}{|c|c|}
\hline Mesh & $\frac{\left\|u-u^{h}\right\|_{0, \Omega}}{\|u\|_{0, \Omega}}$ \\
\hline$a$ & $7.4 \times 10^{-16}$ \\
$b$ & $2.3 \times 10^{-15}$ \\
$c$ & $2.6 \times 10^{-15}$ \\
$d$ & $7.5 \times 10^{-15}$ \\
$e$ & $1.2 \times 10^{-14}$ \\
\hline
\end{tabular}

Table II. Patch test for the VEM.

In Figure 5, we compare the errors obtained by using the VEM and the isoparametric bilinear FEM with $2 \times 2$ Gauss quadrature (labeled ISO-Q1). The right-hand-side $f$ and the boundary data $g$ are defined according to the exact solution $u(x, y)=\sin (2 x) \sin (3 y)+\log (2+x y)$. Relative errors in the $L^{2}$ norm and in the $H^{1}$ seminorm for VEM and bilinear FEM are compared; we observe that both methods deliver the optimal convergence rates and achieve comparable accuracy. Hereafter, we only show errors in the $L^{2}$ norm; errors in the $H^{1}$ seminorm behave as expected.

In Figure 6, we show the robustness of the VEM, that is, the sensitivity of the VEM with respect to the parameter $\tau$ by setting $\boldsymbol{S}=\tau \boldsymbol{I}$ in (57); the parameter $\tau$ can be viewed as the energy of the function $\Psi$. We observe that the VEM is very robust with respect to the choice of $\tau$ : very good accuracy is realized for values of $\tau$ that range from 1 to $10^{3}$. In the robustness study, $\tau<1$ is not considered because, for the chosen problem, the consistency Copyright (c) 2014 John Wiley \& Sons, Ltd.

Int. J. Numer. Meth. Engng 2014; 1:1-33 Prepared using nmeauth.cls 


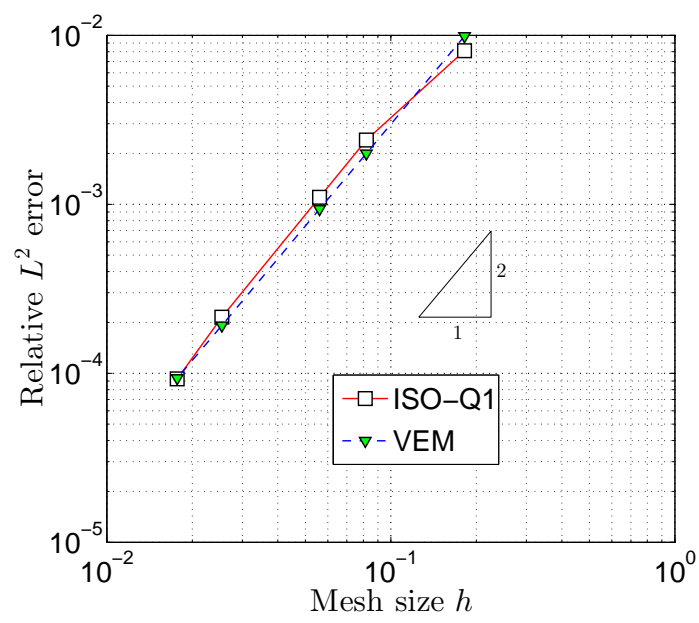

(a)

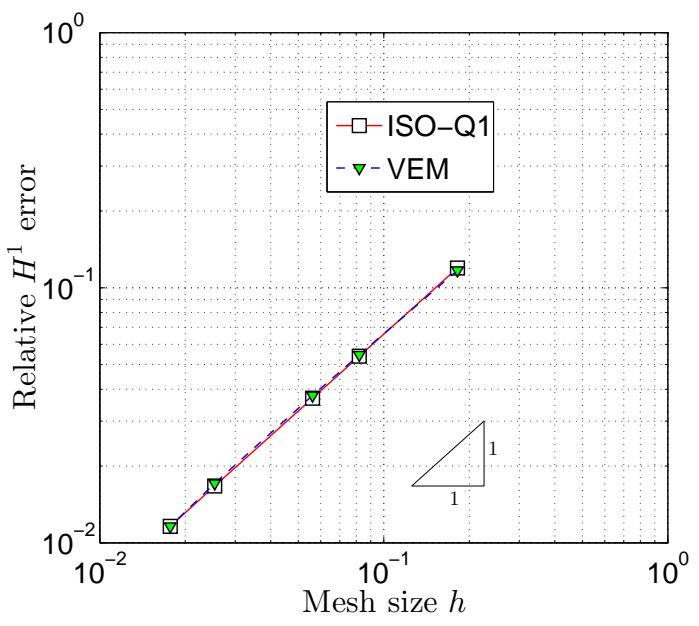

(b)

Figure 5. Rate of convergence of FEM and VEM for the 2D Poisson problem. (a) $L^{2}$ norm; and (b)

$$
H^{1} \text { seminorm. }
$$

matrix alone is sufficient for the convergence of the method. This fact is not at odds with the theory, as the global stiffness matrix can be nonsingular even if the local stiffness matrices are rank-deficient: the singularity is avoided in this particular case due to the smooth Dirichlet boundary conditions.

To more closely examine the role of the stability correction for the Poisson problem, we choose an exact solution that is discontinuous at the boundary as the one depicted in Figure 7. This solves the boundary-value problem in (1) with $f=0$ and piecewise-constant Dirichlet boundary condition. In Figure 7, the VEM solution for $\tau=1$ is plotted. In Figure 8, we present the VEM solution obtained on the second mesh for decreasing $\tau$. We notice the appearance of hourglass instabilities, even though the scheme does not become singular for $\tau=0$.

Copyright (C) 2014 John Wiley \& Sons, Ltd.

Int. J. Numer. Meth. Engng 2014; 1:1-33

Prepared using nmeauth.cls 


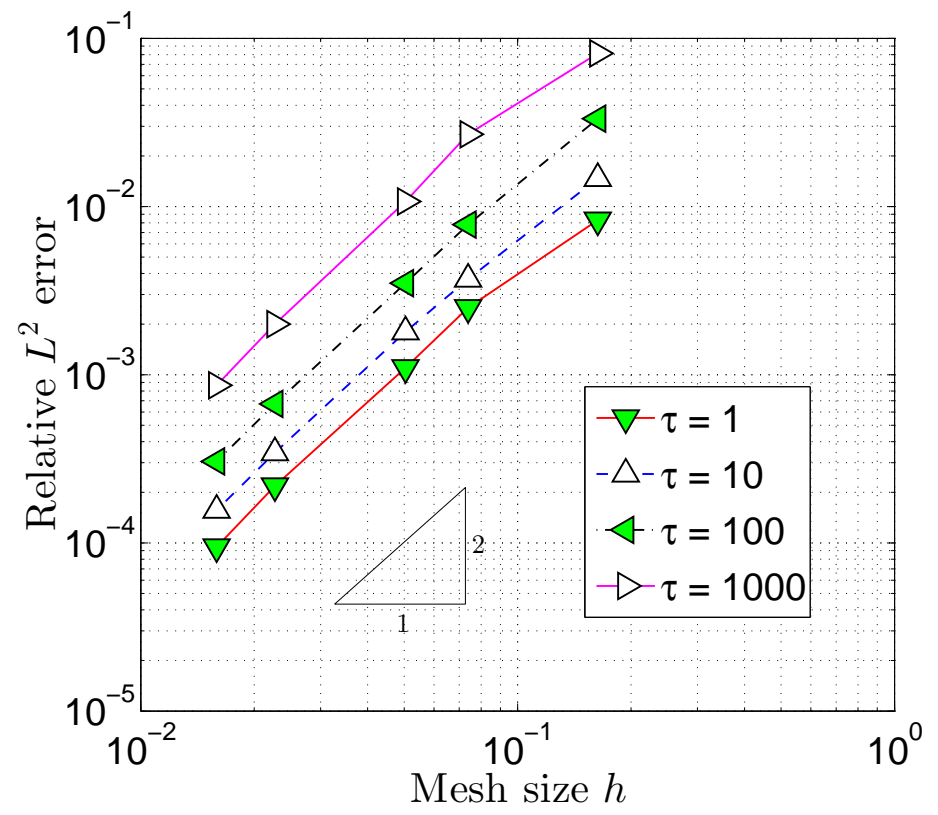

Figure 6. VEM solution for $\boldsymbol{S}=\tau \boldsymbol{I}$, with $\tau=1,10,100,1000$.

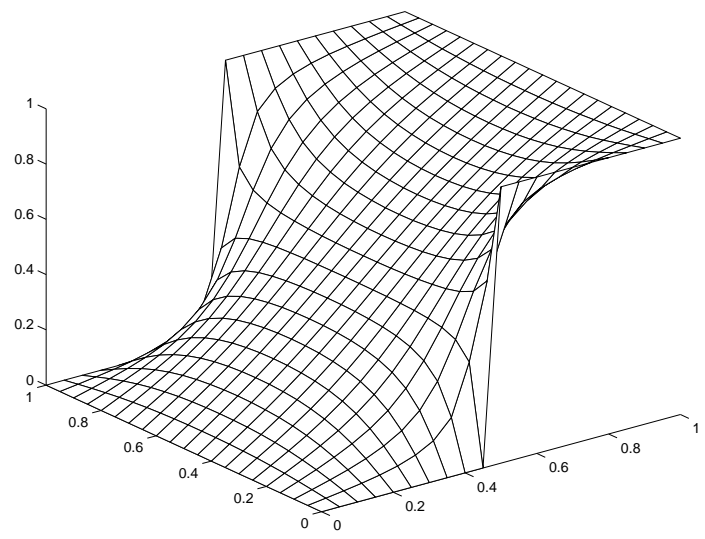

(a)

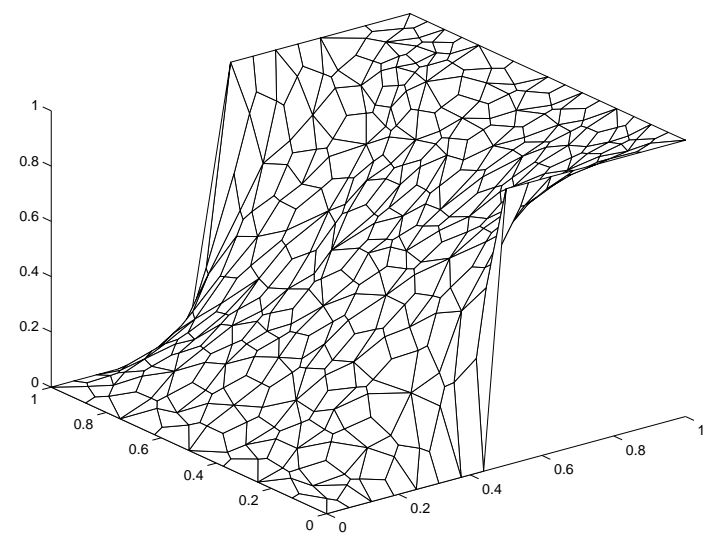

(b)

Figure 7. Laplace problem with discontinuous Dirichlet boundary conditions. (a) Exact solution and (b) VEM solution for $\tau=1$. 


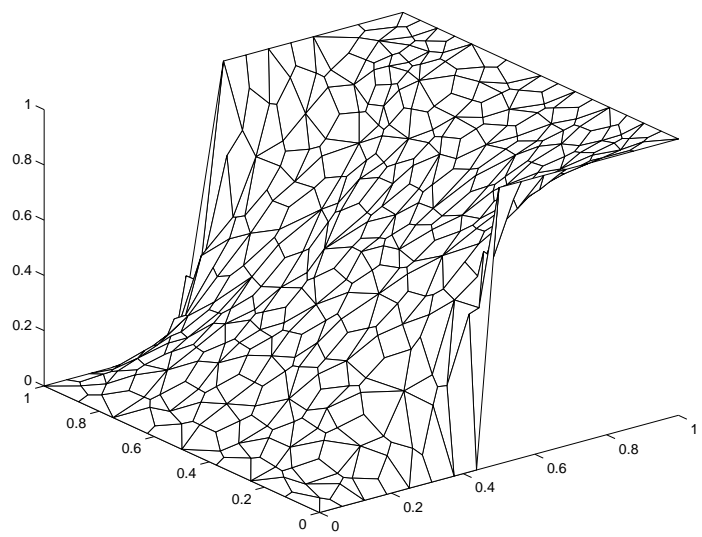

(a)

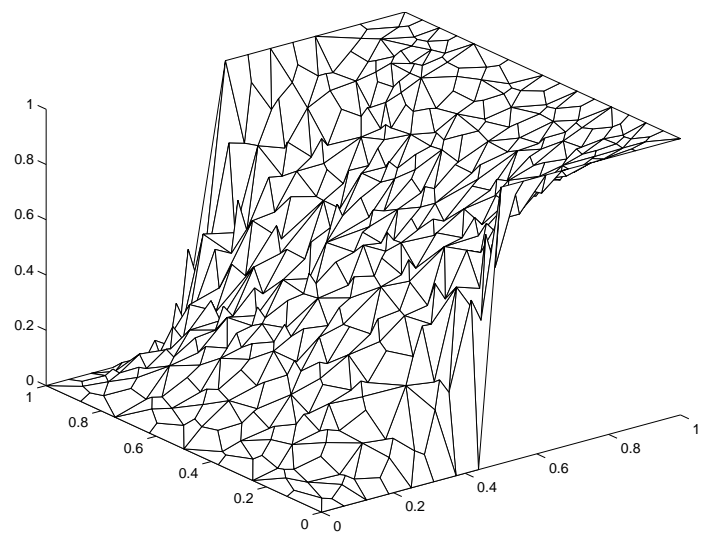

(c)

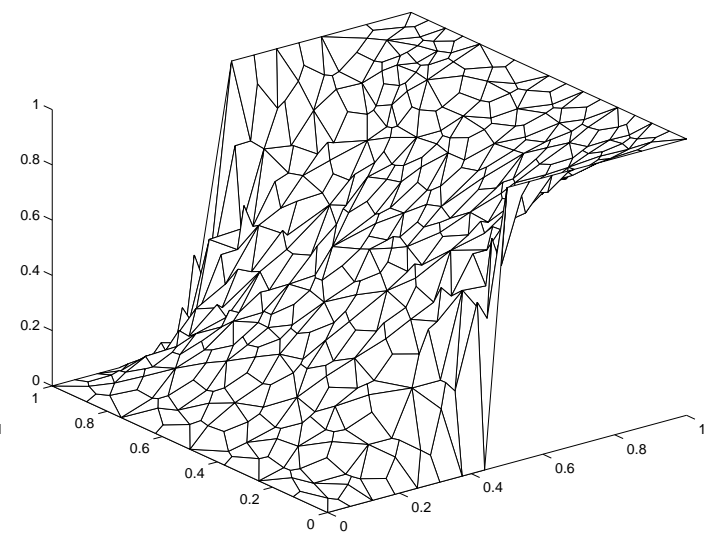

(b)

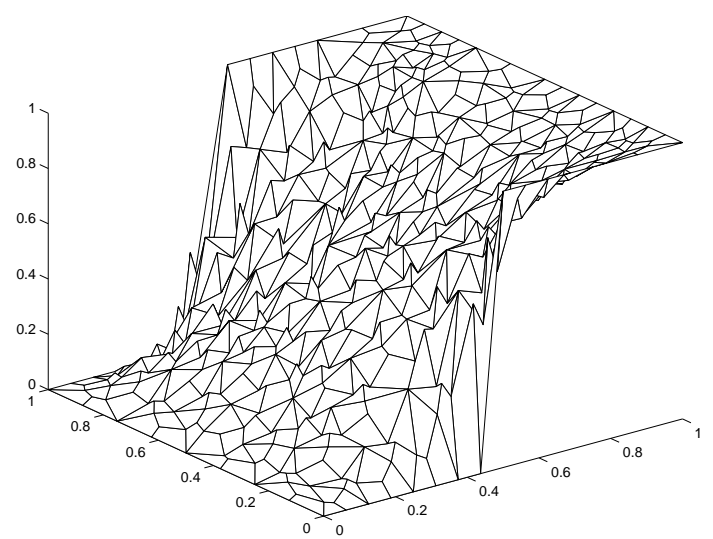

(d)

Figure 8. VEM solutions with $\boldsymbol{S}=\tau \boldsymbol{I}$ for Laplace problem with discontinuous Dirichlet boundary conditions. (a) $\tau=10^{-1}$; (b) $\tau=10^{-2}$; (c) $\tau=10^{-3}$; and (d) $\tau=0$.

10.1.2. Concave quadrilateral meshes and polygonal meshes. The isoparametric bilinear finite element is restricted to convex quadrilateral meshes, because on concave quadrilateral elements, the isoparametric transformation $\boldsymbol{F}$ becomes singular at some point inside the element. No such restrictions are imposed on VEM. As shown in Section 7, given a polygon, not necessarily Copyright (C) 2014 John Wiley \& Sons, Ltd. Int. J. Numer. Meth. Engng 2014; 1:1-33 Prepared using nmeauth.cls 


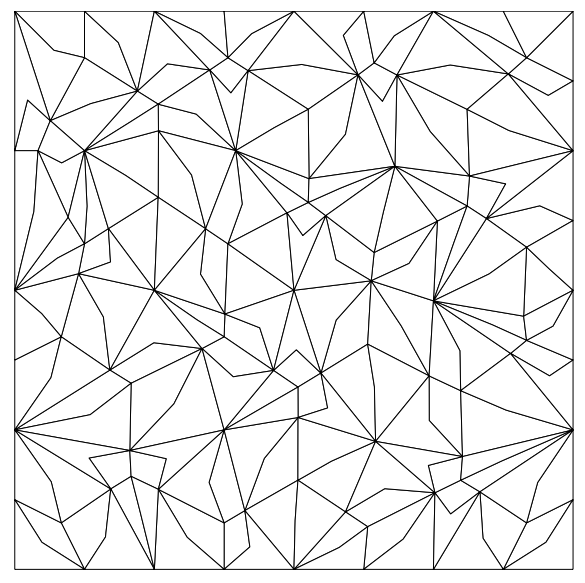

(a)

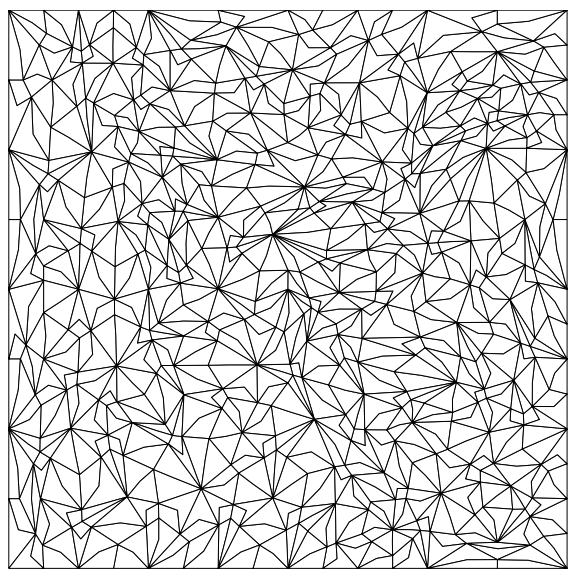

(b)

Figure 9. (a) First and (b) second concave quadrilateral meshes of the sequence.

convex, we can define a finite element space directly on the physical element and compute using (46)-(49) the VEM stability and consistency matrices.

To demonstrate the behavior of the VEM for concave quadrilaterals, we repeat the test shown in Figure 5 on the series of meshes obtained from the previous one by subdividing each quadrilateral into two smaller quadrilaterals, one of which is necessarily concave. The first two meshes of this new sequence are shown in Figure 9. Convergence curves are presented in Figure 10 showing that the error norms are almost identical to those obtained on convex meshes (see Figure 5).

Finally, we report on a numerical experiment for the virtual element decomposition when applied to the finite element space of generalized barycentric coordinates on polygons as discussed in Section 7. We consider a sequence of very irregular Voronoi polygonal meshes with random seed points; in Figure 11, we show the first and second meshes of the sequence. Copyright (C) 2014 John Wiley \& Sons, Ltd. 


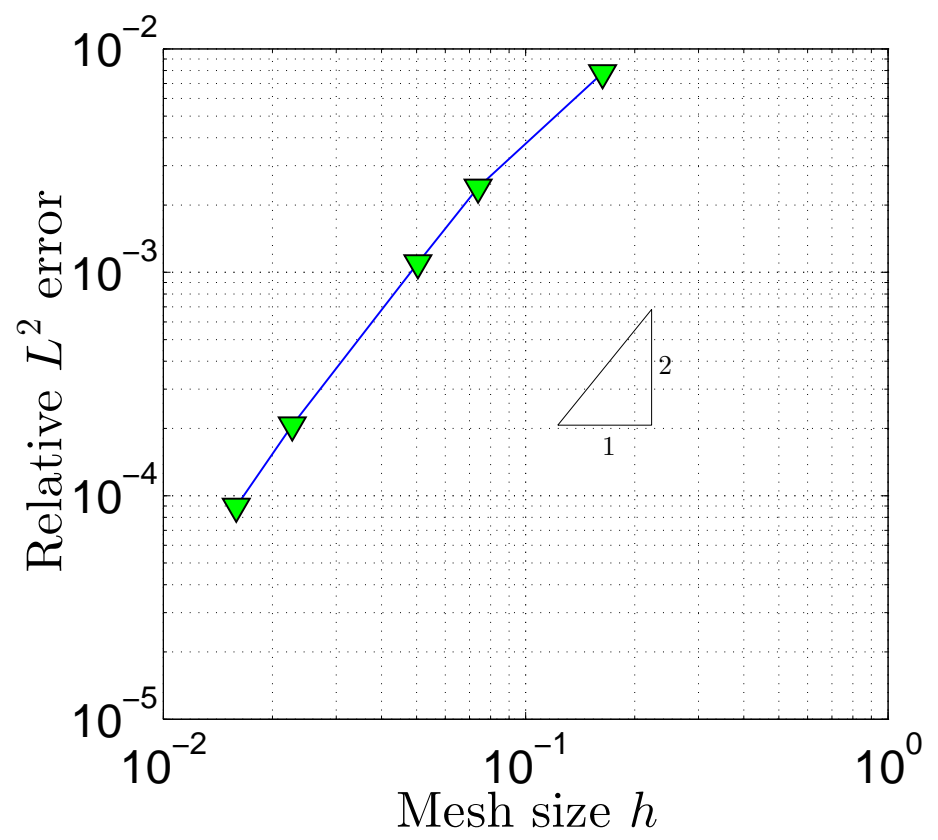

Figure 10. Convergence of VEM for the 2D Poisson problem on the concave meshes.

In Figure 12, we present for the purpose of comparison the relative $L^{2}$ errors on the quadrilateral (convex and concave) and polygonal meshes, revealing that in the VEM the error depends as expected on the mesh size, but does not depend on the shape of the elements.

\subsection{Three-dimensional problems}

In the numerical experiments presented here, we consider three methods:

1. virtual element method (labeled VEM), which corresponds to the stiffness matrix $\boldsymbol{K}^{\mathrm{VEM}}=\boldsymbol{K}_{\mathrm{C}}^{\mathrm{VEM}}+\boldsymbol{K}_{\mathrm{S}}^{\mathrm{VEM}}$, where $\boldsymbol{K}_{\mathrm{C}}^{\mathrm{VEM}}$ and $\boldsymbol{K}_{\mathrm{S}}^{\mathrm{VEM}}$ are defined in (64) and (49), respectively, with $\boldsymbol{S}=h_{e} \boldsymbol{I} ;$ for the irregular hexahedral meshes, we use $h_{e}=\left|\Omega_{e}\right|^{1 / 3}$.

Copyright (c) 2014 John Wiley \& Sons, Ltd.

Int. J. Numer. Meth. Engng 2014; 1:1-33

Prepared using nmeauth.cls 


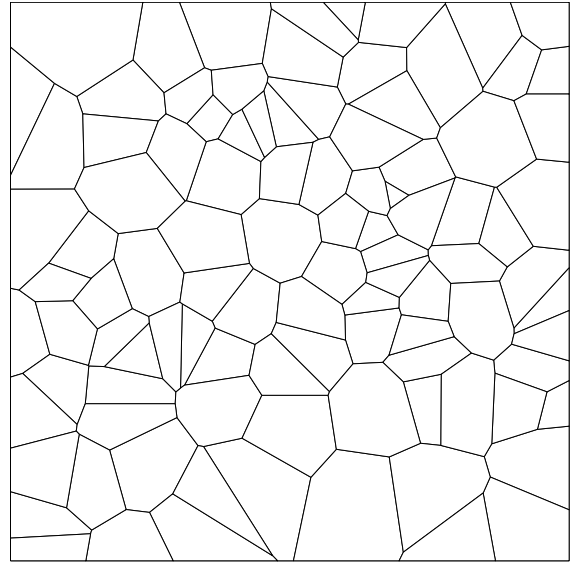

(a)

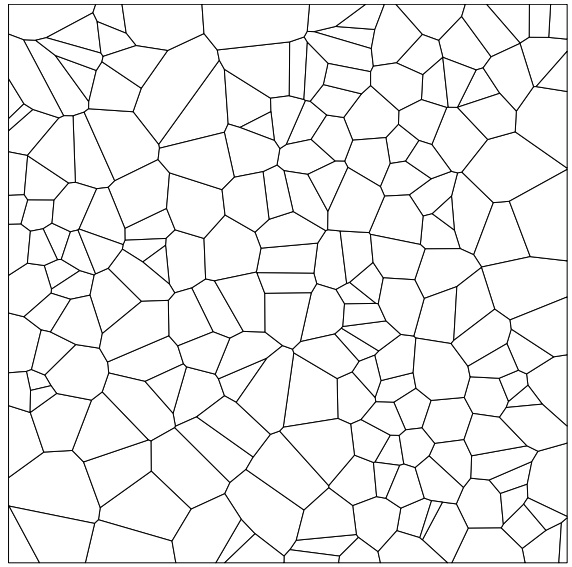

(b)

Figure 11. (a) First and (b) second polygonal meshes of the sequence.

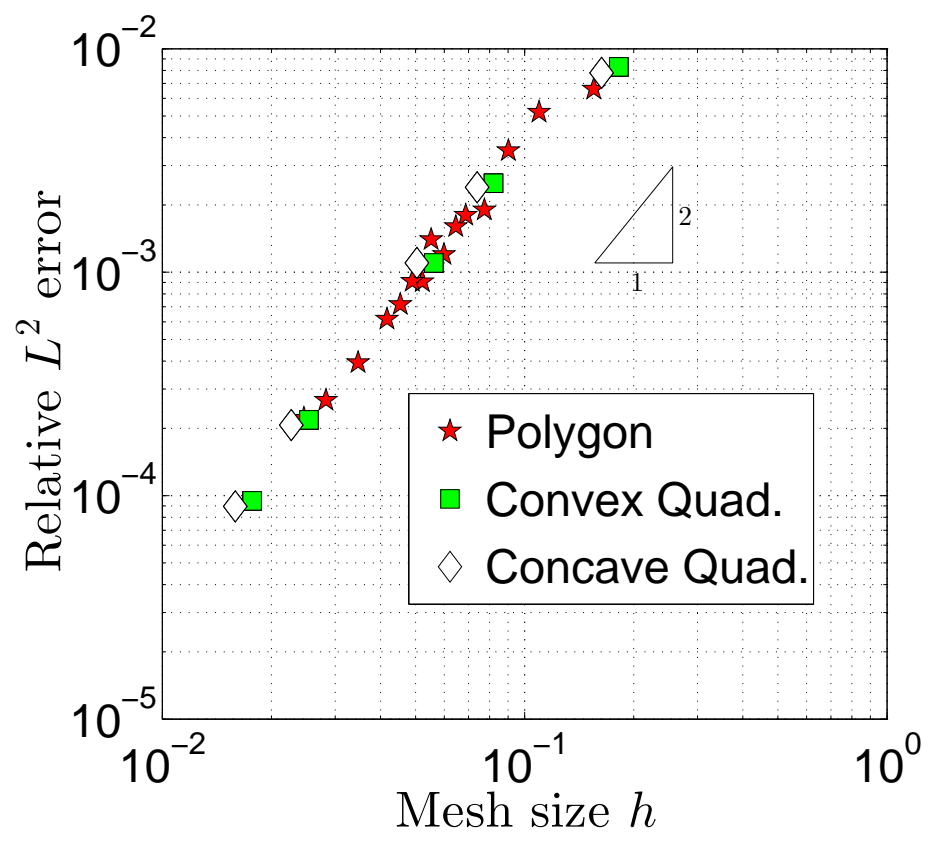

Figure 12. Relative $L^{2}$ error for Poisson problem on polygonal and quadrilateral meshes. 
2. Flanagan-Belytschko scheme (labeled FB), which corresponds to the stiffness matrix $\boldsymbol{K}^{\mathrm{FB}}=\boldsymbol{K}_{\mathrm{C}}^{\mathrm{FB}}+\boldsymbol{K}_{\mathrm{s}}^{\mathrm{FB}}$, where $\boldsymbol{K}_{\mathrm{C}}^{\mathrm{FB}}$ and $\boldsymbol{K}_{\mathrm{s}}^{\mathrm{FB}}$ are defined in (13) and (25), respectively. We set $\bar{\epsilon}=\left|\Omega_{e}\right|^{-2 / 3}$ in $\boldsymbol{K}_{\mathrm{S}}^{\mathrm{FB}}$ to obtain the correct scaling. Note that $\boldsymbol{K}_{\mathrm{C}}^{\mathrm{VEM}}=\boldsymbol{K}_{\mathrm{C}}^{\mathrm{FB}}$.

3. standard isoparametric finite elements with $2 \times 2 \times 2$ Gauss quadrature on the reference biunit cube (labeled ISO-Q1).

Consider the domain $\Omega=(0,1)^{3}$, which is discretized by a sequence of four unstructured meshes of decreasing mesh size. Each mesh consists of subdivisions of the unit cube into highly irregular hexahedra with curved faces. In Table III, the mesh parameters are listed, and the first two meshes of the sequence are depicted in Figure 13.

\begin{tabular}{|c|c|c|c|}
\hline Mesh & $\begin{array}{c}\text { Number of } \\
\text { elements }\end{array}$ & $\begin{array}{c}\text { Number of } \\
\text { nodes }\end{array}$ & $\begin{array}{c}\text { Element } \\
\text { diameter } h\end{array}$ \\
\hline$a$ & 144 & 243 & $4.2 \times 10^{-1}$ \\
$b$ & 8952 & 10985 & $1.2 \times 10^{-2}$ \\
$c$ & 18580 & 22365 & $9.3 \times 10^{-2}$ \\
$d$ & 82488 & 95079 & $5.5 \times 10^{-2}$ \\
\hline
\end{tabular}

Table III. Mesh parameters for the unstructured hexahedral meshes.

10.2.1. Patch test First, we show that all three methods pass the patch test. Consider the nonhomogeneous Dirichlet boundary condition $g(\boldsymbol{x})=1-2 x+3 y-4 z$ in (1) with $f=0$ so that $u(\boldsymbol{x})=g(\boldsymbol{x})$ is the exact solution. The relative $L^{2}$ error norms for the three different methods are listed in Table IV; these results show that all three methods pass the patch test. 


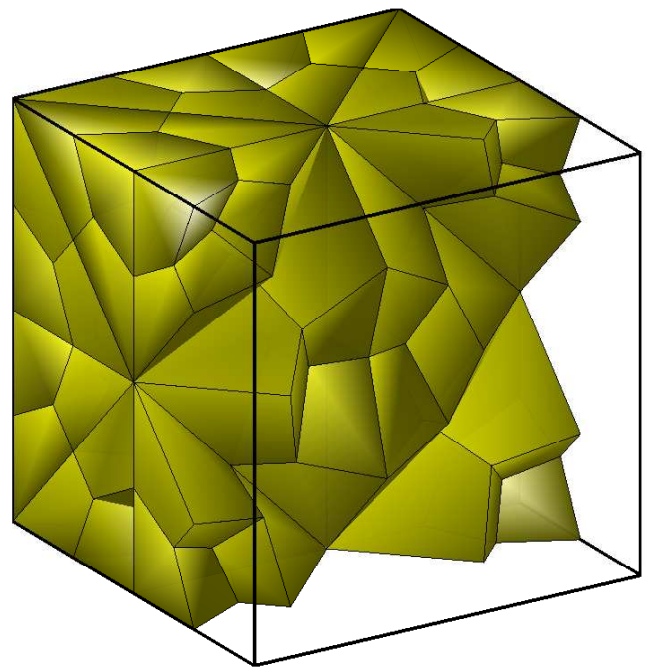

(a)

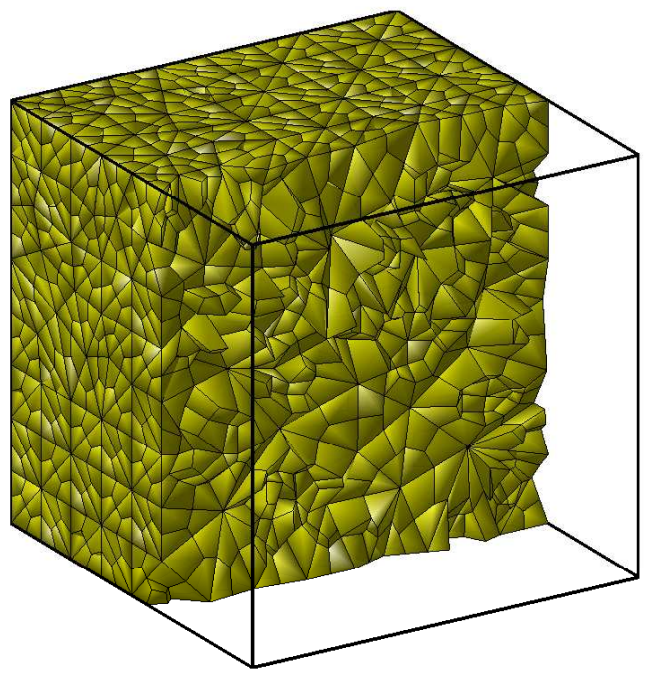

(b)

Figure 13. Meshes (a) and (b) in the sequence of unstructured hexahedral meshes.

\begin{tabular}{|c|c|c|c|}
\hline \multirow{2}{*}{ Mesh } & \multicolumn{3}{|c|}{$\frac{\left\|u-u^{h}\right\|_{0, \Omega}}{\|u\|_{0, \Omega}}$} \\
\cline { 2 - 4 } & ISO-Q1 & FB [8] & VEM \\
\hline$a$ & $2.6 \times 10^{-16}$ & $2.7 \times 10^{-16}$ & $2.9 \times 10^{-16}$ \\
$b$ & $8.1 \times 10^{-15}$ & $2.5 \times 10^{-15}$ & $2.0 \times 10^{-15}$ \\
$c$ & $5.2 \times 10^{-16}$ & $4.0 \times 10^{-15}$ & $3.2 \times 10^{-15}$ \\
$d$ & $5.9 \times 10^{-16}$ & $1.0 \times 10^{-14}$ & $8.7 \times 10^{-15}$ \\
\hline
\end{tabular}

Table IV. Relative $L^{2}$ error norms for the patch test in $3 \mathrm{D}$.

10.2.2. Comparison of the methods We consider the Poisson problem with the right-hand-side $f$ and the Dirichlet boundary value $g$ chosen so that $u(x, y, z)=\sin (x y) \cos (y z)+x^{3} z-e^{x-3 y-z}$ is the exact solution. In Figure 14, the relative $L^{2}$ and $H^{1}$ error curves for the three methods Copyright (c) 2014 John Wiley \& Sons, Ltd. Int. J. Numer. Meth. Engng 2014; 1:1-33 Prepared using nmeauth.cls 
are compared; we observe that all three methods behave very similarly.

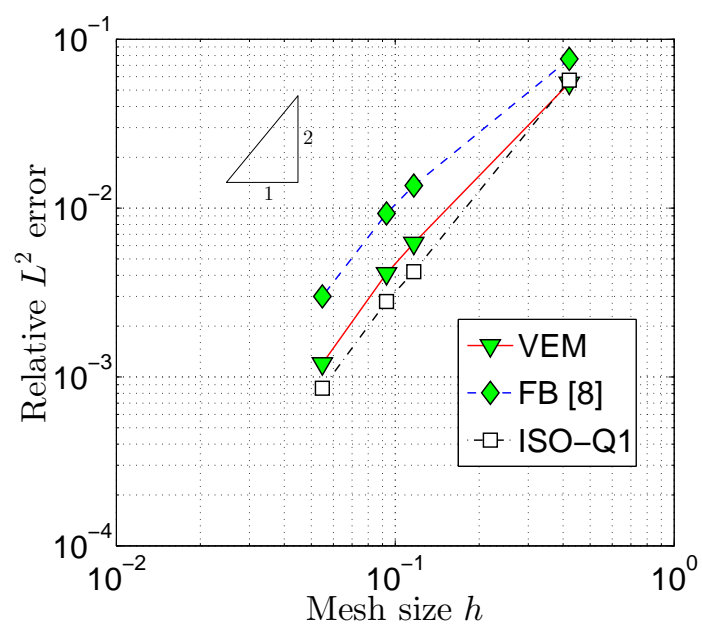

(a)

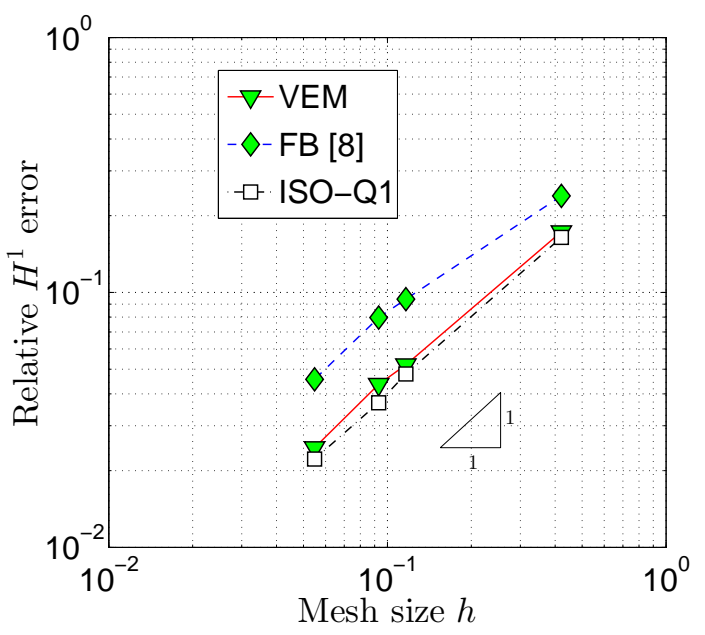

(b)

Figure 14. Rate of convergence of FEM and VEM for the 3D Poisson problem. (a) $L^{2}$ norm; and (b)

$$
H^{1} \text { seminorm. }
$$

The next numerical experiment is presented to demonstrate that taking $\boldsymbol{S}=h_{e} \boldsymbol{I}$ is the correct scaling for the Poisson problem in three dimensions. To observe the effect of scaling with respect to the matrix $\boldsymbol{S}$, fine meshes are needed. Instead, we achieve the same end by scaling the domain by a factor of $10^{-2}$. Then, we consider the VEM with $\boldsymbol{S}=\boldsymbol{I}$. In Figure 15 , plots of the relative error norms are shown, and we clearly observe that the badly-scaled VEM does not converge.

Lastly, the effect of scaling $\boldsymbol{S}$ by a very small quantity is studied. As in the two-dimensional case, we consider a Poisson problem with discontinuous Dirichlet boundary conditions, which activates the spurious zero-energy modes. In (1), we set $f=0$, and $g(x, y)=1$ for $x<0.5$ and $g(x, y)=0$ for $x>0.5$ (see Figure 16). We discretize the biunit cube uniformly into smaller cubes of size $h_{e}=1 / 32$, and plot the solutions on the planes $z=2 h=0.0625$ and Copyright (C) 2014 John Wiley \& Sons, Ltd. 


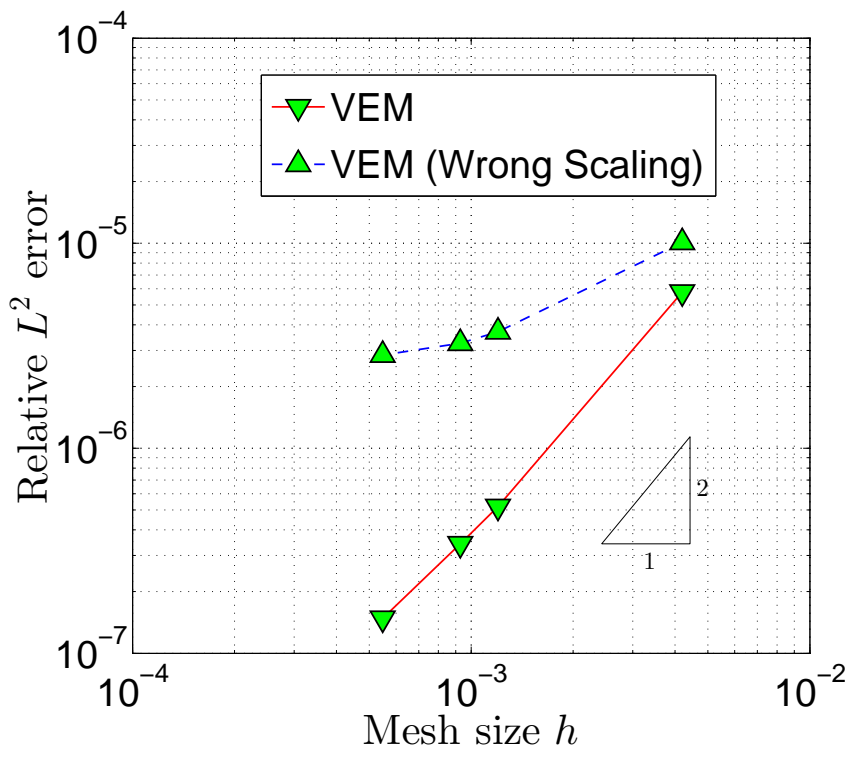

Figure 15. Relative $L^{2}$ error for 3D Poisson problem using different choices of the scaling.

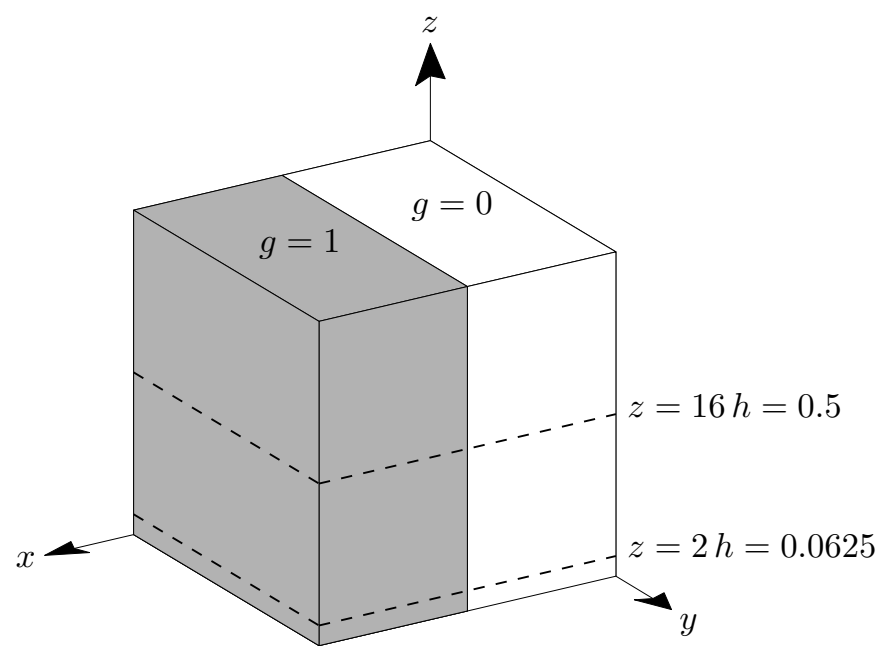

Figure 16. Laplace problem in 3D with discontinuous Dirichlet boundary conditions.

$z=16 h=0.5$. First, we select the correctly-scaled VEM stability matrix; that is, $\boldsymbol{S}=h_{e} \boldsymbol{I}$, Copyright (c) 2014 John Wiley \& Sons, Ltd. Int. J. Numer. Meth. Engng 2014; 1:1-33

Prepared using nmeauth.cls 


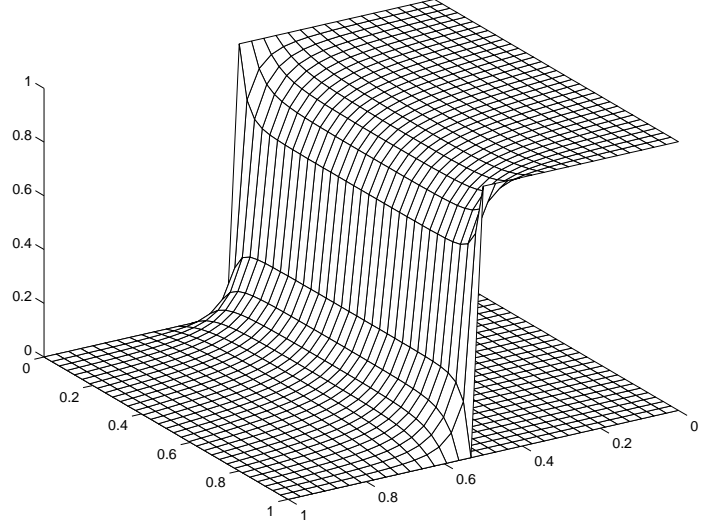

(a)

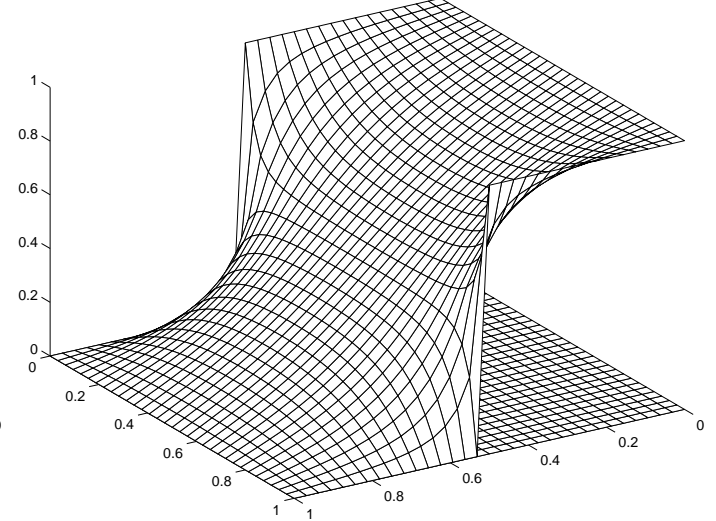

(b)

Figure 17. Virtual element solution for $\boldsymbol{S}=h \boldsymbol{I}$. (a) $z=0.0625$; and (b) $z=0.5$.

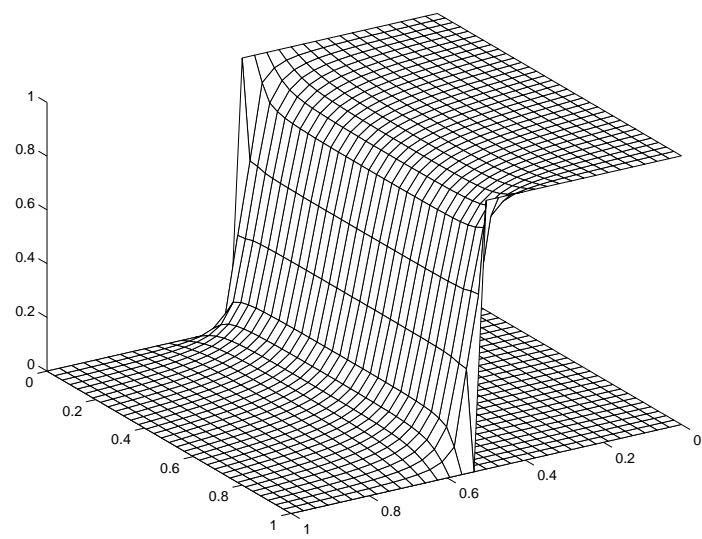

(a)

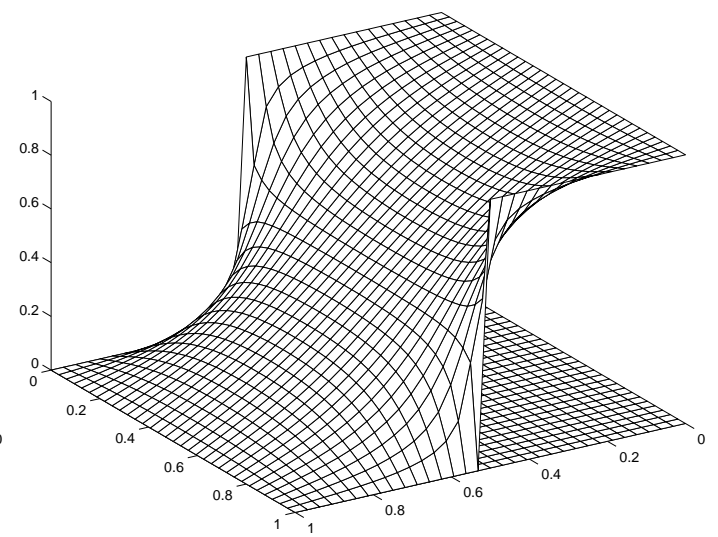

(b)

Figure 18. Isoparametric FE solution. (a) $z=0.0625$; and (b) $z=0.5$.

whose solution is shown in Figure 17. This solution is indistinguishable when compared to the solution obtained by the ISO-Q1 method (Figure 18). Next, we present results when the VEM stability term is very small. We choose $\boldsymbol{S}=10^{-3} \boldsymbol{I}$ in Figure 19 and $\boldsymbol{S}=\mathbf{0}$ in Figure 20. We observe that when $\boldsymbol{S}=10^{-3} \boldsymbol{I}$ oscillations start to develop in the numerical solution, which degenerates for $\boldsymbol{S}=\mathbf{0}$. On the contrary, when $\boldsymbol{S}=h_{e} \boldsymbol{I}=3.125 \times 10^{-2} \boldsymbol{I}$ the solution is not Copyright (C) 2014 John Wiley \& Sons, Ltd. 


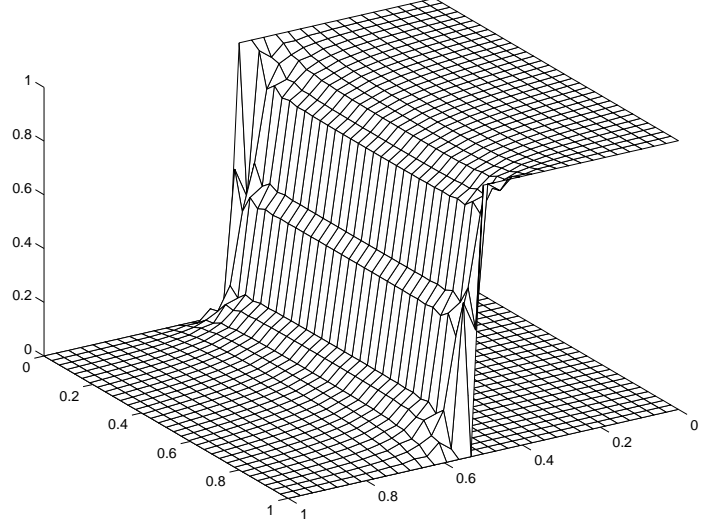

(a)

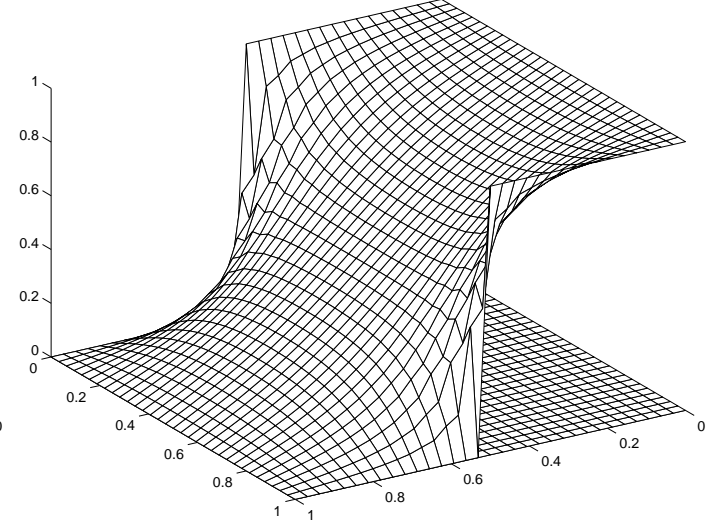

(b)

Figure 19. Virtual element solution for $\boldsymbol{S}=10^{-3} \boldsymbol{I}$. (a) $z=0.0625$; and (b) $z=0.5$.

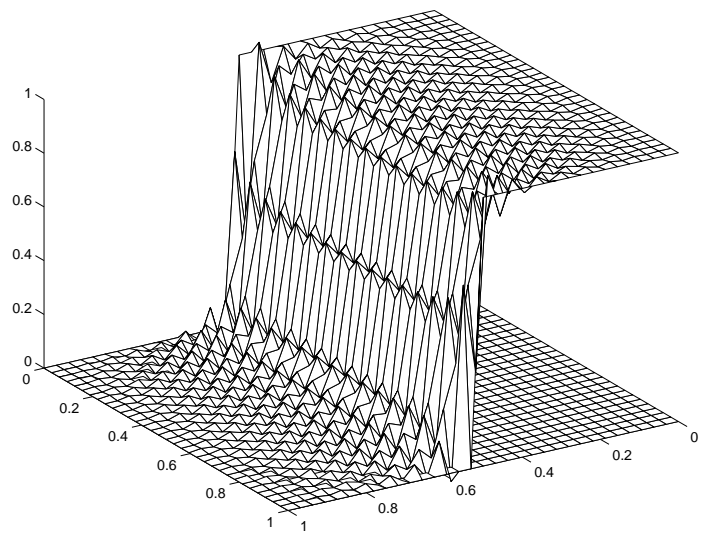

(a)

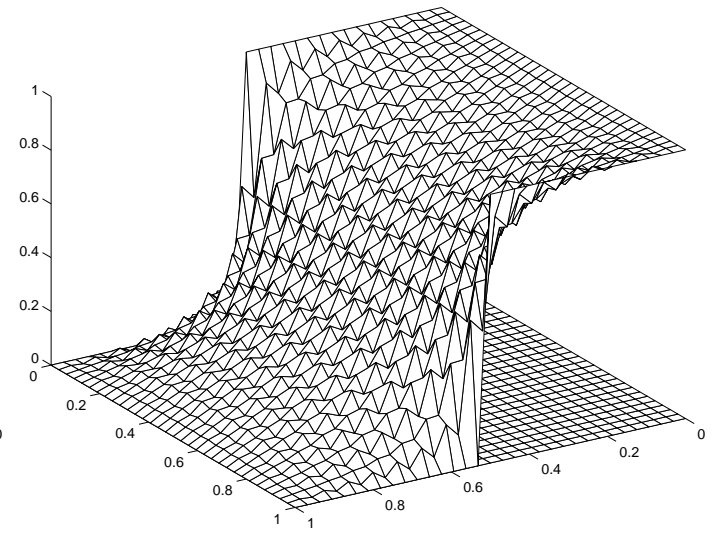

(b)

Figure 20. Virtual element solution for $\boldsymbol{S}=\mathbf{0}$. (a) $z=0.0625$; and (b) $z=0.5$.

oscillatory. The VEM solution also degrades when $\boldsymbol{S}$ is too large. In Figure 21, the VEM solution for $\boldsymbol{S}=\boldsymbol{I}$ is shown.

Copyright (C) 2014 John Wiley \& Sons, Ltd.

Int. J. Numer. Meth. Engng 2014; 1:1-33

Prepared using nmeauth.cls 


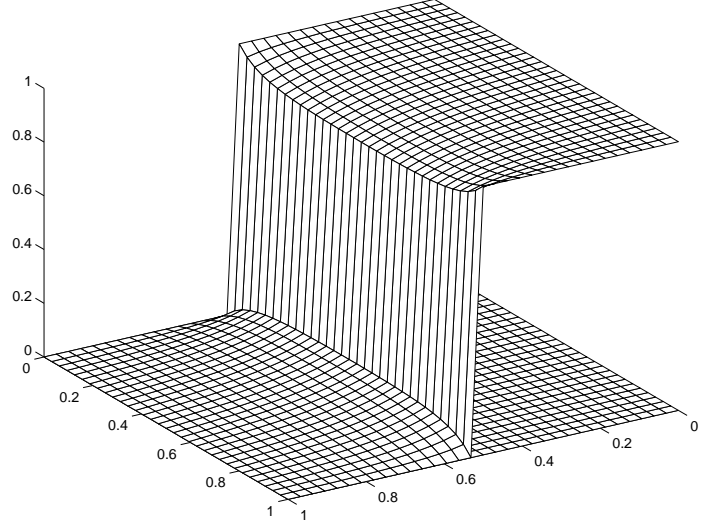

(a)

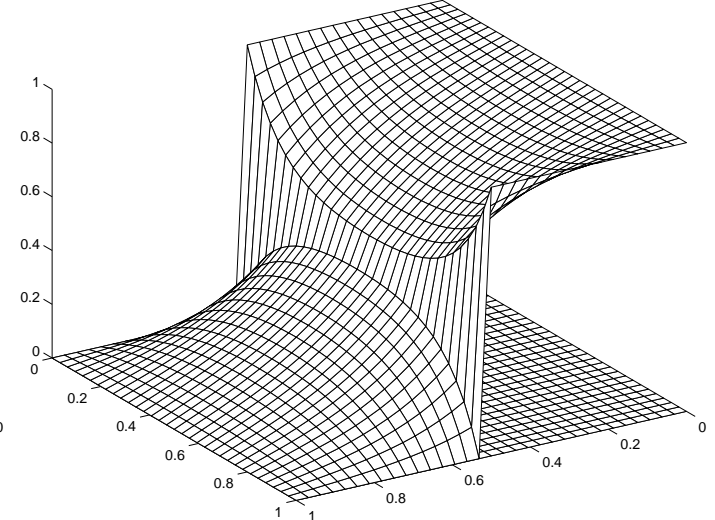

(b)

Figure 21. Virtual element solution for $\boldsymbol{S}=\boldsymbol{I}$. (a) $z=0.0625$; and (b) $z=0$.

\section{CONCLUDING REMARKS}

In this paper, we showed that the virtual element method (VEM) [16] can be viewed as a general stabilized underintegrated Galerkin method. We presented a first-order virtual element approach that was based on isoparametric elements for the Poisson problem in two- and three-dimensions, and established quantitative connections of this approach with well-known hourglass stabilization techniques for four-node quadrilateral and eight-node hexahedral finite elements [8-10].

The robustness and flexibility of the VEM was demonstrated via numerical experiments on quadrilateral, polygonal (convex and concave elements), and hexahedral meshes. The importance of the choice and selection of the stability parameter $\tau$ was emphasized, and for suitable choices for $\tau$, optimal convergence rates of the VEM in the $L^{2}$ norm and the $H^{1}$ seminorm of 2 and 1 , respectively, were realized. These observations indicate that the virtual element method provides a systematic and mathematically rigorous approach to develop 
stable underintegrated finite element methods. Two open-issues that persist with hourglass control finite element methods are lack of a convergence theory, and suitable choices for the stabilization parameter $\tau$ [10]. The VEM gives definitive answers to these questions: convergence theory is now available [16] and the theory provides clear guidelines on selecting $\tau$ [23]. Furthermore, the theory is applicable to $C^{0}$ arbitrary-order polygonal and polyhedral elements [16], and also to elements that possess arbitrary regularity [26, 27]. Hence, in such general settings, the virtual element method affords the development of stable and computationally efficient Galerkin schemes.

In this paper, hourglass stabilization for the Poisson equation was the focus. Recent studies on the VEM have examined elasticity [28, 29] and plate bending [26]. For vectorial problems, the number of spurious singular (hourglass) modes substantially increases, which likely makes it more difficult to draw direct links between VEM and prior work on hourglass control techniques using the FEM. Further investigations of the VEM for broader classes of partial differential equations, and in particular for linear and nonlinear solid continua are fruitful directions for future research.

\section{APPENDIX A}

Isoparametric finite elements and the patch test

It is well-known that the bilinear and trilinear isoparametric elements pass the patch test. In this section, we examine the implications of using a quadrature formula and its effect on passing or failing the patch test. A similar analysis for the four-node quadrilateral is performed by Talischi and Paulino [20].

Copyright (c) 2014 John Wiley \& Sons, Ltd.

Int. J. Numer. Meth. Engng 2014; 1:1-33

Prepared using nmeauth.cls 
As stated in Theorem 1, a sufficient condition for passing the patch test is that the method is consistent and stable. Consistency means that the quantity

$$
\int_{\Omega_{e}} \nabla v_{h} \cdot \nabla p_{1} \mathrm{~d} \boldsymbol{x}, \quad v_{h} \in \mathbb{Q}_{1}\left(\Omega_{e}\right), \quad p_{1} \text { is a linear polynomial }
$$

is exactly computed. The VEM does so by construction; let us see what happens when we use a quadrature formula on the reference element. For simplicity, take $v_{h}=\phi_{i}, p_{1}=x$. On mapping back to the reference element, the exact element stiffness matrix can be written as

$$
\int_{\Omega_{e}} \nabla \phi_{i} \cdot \nabla x \mathrm{~d} \boldsymbol{x}=\int_{\Omega_{0}} \boldsymbol{J}_{\boldsymbol{F}}^{-\mathrm{T}}\left(\nabla_{\boldsymbol{\xi}} N_{i}\right) \cdot \boldsymbol{J}_{\boldsymbol{F}}^{-\mathrm{T}}\left(\nabla_{\boldsymbol{\xi}} x\right) \operatorname{det}\left(\boldsymbol{J}_{\boldsymbol{F}}\right) \mathrm{d} \boldsymbol{\xi}
$$

which is approximated using a quadrature formula. Now, the function $x$ when seen on the reference element becomes $x \circ \boldsymbol{F}$, that is, the first component $F_{1}$ of the map $\boldsymbol{F}$. It is verified that

$$
\boldsymbol{J}_{\boldsymbol{F}}^{-\mathrm{T}}\left(\nabla_{\boldsymbol{\xi}} F_{1}\right)= \begin{cases}{\left[\begin{array}{lll}
1 & 0 & 0
\end{array}\right]^{\mathrm{T}}} & \text { in } 3 \mathrm{D}, \\
{\left[\begin{array}{ll}
1 & 0
\end{array}\right]^{\mathrm{T}}} & \text { in } 2 \mathrm{D},\end{cases}
$$

so that

$$
\begin{aligned}
\boldsymbol{J}_{\boldsymbol{F}}^{-\mathrm{T}}\left(\nabla_{\boldsymbol{\xi}} N_{i}\right) \cdot \boldsymbol{J}_{\boldsymbol{F}}^{-\mathrm{T}}\left(\nabla_{\boldsymbol{\xi}} F_{1}\right) \operatorname{det}\left(\boldsymbol{J}_{\boldsymbol{F}}\right) & =\text { first component of } \boldsymbol{J}_{\boldsymbol{F}}^{-\mathrm{T}}\left(\nabla_{\boldsymbol{\xi}} N_{i}\right) \operatorname{det}\left(\boldsymbol{J}_{\boldsymbol{F}}\right) \\
& =\text { first component of } \operatorname{cof}\left(\boldsymbol{J}_{\boldsymbol{F}}\right)\left(\nabla_{\boldsymbol{\xi}} N_{i}\right)
\end{aligned}
$$

where $\operatorname{cof}\left(\boldsymbol{J}_{\boldsymbol{F}}\right)$ is the cofactor matrix of $\boldsymbol{J}_{\boldsymbol{F}}$, that is, the matrix such that

$$
\boldsymbol{J}_{\boldsymbol{F}}^{-1}=\frac{1}{\operatorname{det}\left(\boldsymbol{J}_{\boldsymbol{F}}\right)} \operatorname{cof}\left(\boldsymbol{J}_{\boldsymbol{F}}\right)^{\mathrm{T}}
$$

Hence, to verify that the method is consistent, we need to check if $\operatorname{cof}\left(\boldsymbol{J}_{\boldsymbol{F}}\right)\left(\nabla_{\boldsymbol{\xi}} N_{i}\right)$ is exactly integrated by the chosen quadrature formula. It is clear that $\operatorname{cof}\left(\boldsymbol{J}_{\boldsymbol{F}}\right)\left(\nabla_{\boldsymbol{\xi}} N_{i}\right)$ has polynomial components; we need to check the degree with respect to each scalar variable.

Copyright (C) 2014 John Wiley \& Sons, Ltd.

Int. J. Numer. Meth. Engng 2014; 1:1-33

Prepared using nmeauth.cls 
- For the four-node quadrilateral element it can be easily seen that $\operatorname{cof}\left(\boldsymbol{J}_{\boldsymbol{F}}\right)\left(\nabla_{\boldsymbol{\xi}} N_{i}\right)$ is linear in each component. Hence, any quadrature formula that exactly integrates functions that are affine in each variable yields a method that is consistent. In fact, we know that the one-point Gauss rule is consistent but unstable, whereas the $2 \times 2$ trapezoidal rule is consistent and stable, and therefore passes the patch test.

- For the eight-node hexahedral element it can be observed that $\operatorname{cof}\left(\boldsymbol{J}_{\boldsymbol{F}}\right)\left(\nabla_{\boldsymbol{\xi}} N_{i}\right)$ is quadratic in each component. Hence, any quadrature formula that exactly integrates functions that are quadratic in each variable yields a method that is consistent. For instance, the one-point Gauss rule is inconsistent and unstable, the $2 \times 2 \times 2$ tensorproduct Gauss rule is consistent and stable and therefore passes the patch test, and the $2 \times 2 \times 2$ trapezoidal rule is inconsistent but stable. For the last case, it can be numerically shown that the patch test is not passed.

\section{APPENDIX B}

\section{B.1. Stencils for the uniform square mesh}

On using the energy $\tau$ of the function $\Psi$ as a parameter, it is possible to compare the VEM stiffness matrix in (61) to well-known finite-difference stencils on simple nodal configurations, and also to fully integrated quadrilateral finite elements. Such comparisons for FEM have been performed in References [9, 10]. For a uniform square mesh, the stencil shown in Figure 22 is

obtained. For a square of side $a$, we have $\tau=\int_{\Omega_{e}}|\nabla \Psi|^{2} \mathrm{~d} \boldsymbol{x}=2 / 3$. With this value of $\tau$, we obtain the scheme corresponding to the exact integration of the four-node isoparametric finite element (see Figure 23). On taking $\tau=2$, we recover the classical 5-point stencil (Figure 24)

Copyright (c) 2014 John Wiley \& Sons, Ltd.

Int. J. Numer. Meth. Engng 2014; 1:1-33

Prepared using nmeauth.cls 

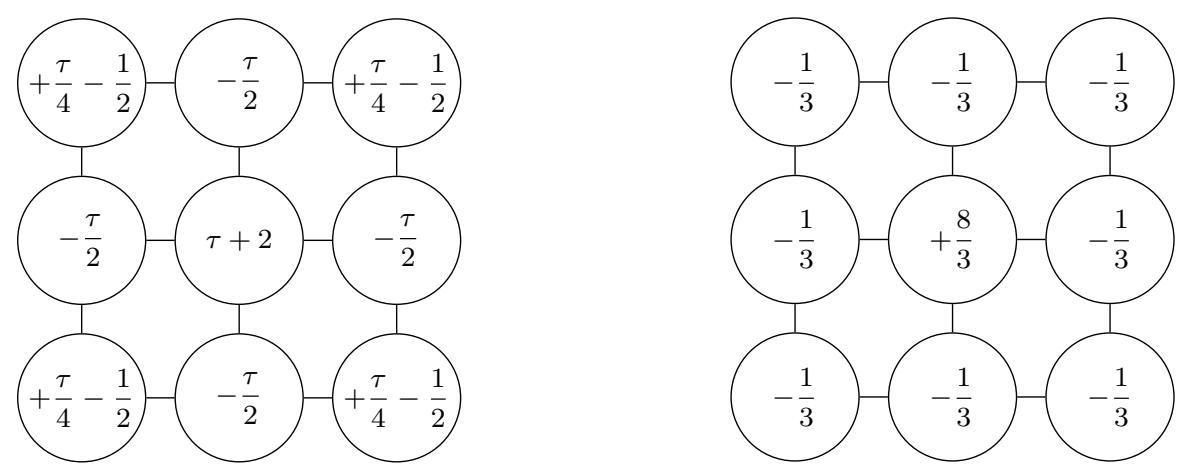

Figure 22. Family of schemes for general $\tau$. Figure 23. $\tau=2 / 3$ is the exact integration of $\mathbb{Q}_{1}\left(\Omega_{e}\right)$.

and for $\tau=4 / 3$, we obtain the fourth-order 9-point stencil (Figure 25). Finally, $\tau=1$ leads
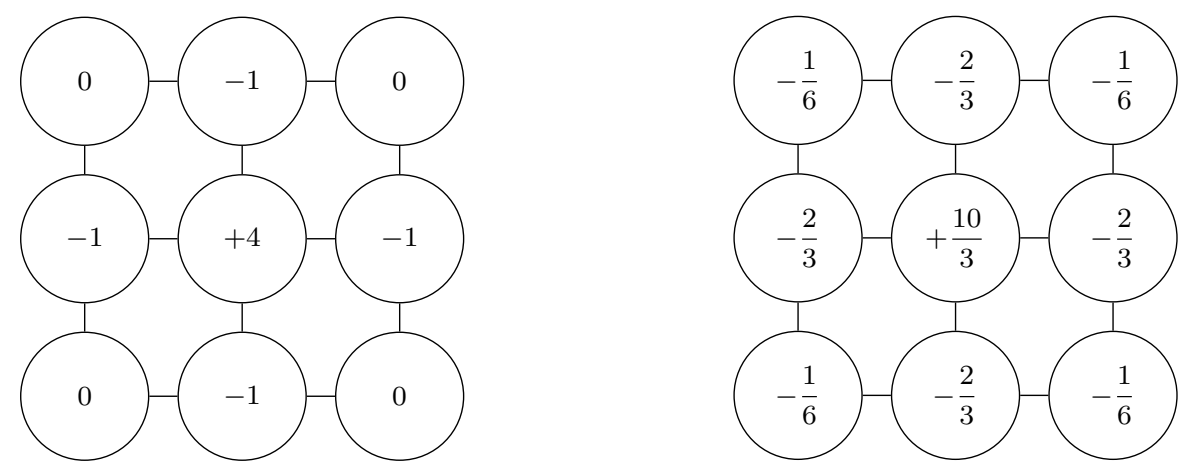

Figure 24. $\tau=2$ is the classical 5-point stencil. Figure 25. $\tau=4 / 3$ is the fourth-order 9-point stencil.

to a virtual element scheme with $\boldsymbol{S}=\boldsymbol{I}$ (Figure 26) and when $\tau=0$, we recover the unstable hourglass stencil shown in Figure 27.

\section{B.2. Consistency-stability decomposition for particular quadrilaterals}

On using (55) and (63), the consistency-stability decompositions for a few particular convex quadrilaterals are presented. All the cases under consideration are parallelograms, and hence the stability matrix is always given by (62). For the consistency matrix, we refer to the Copyright (C) 2014 John Wiley \& Sons, Ltd.

Int. J. Numer. Meth. Engng 2014; 1:1-33

Prepared using nmeauth.cls 

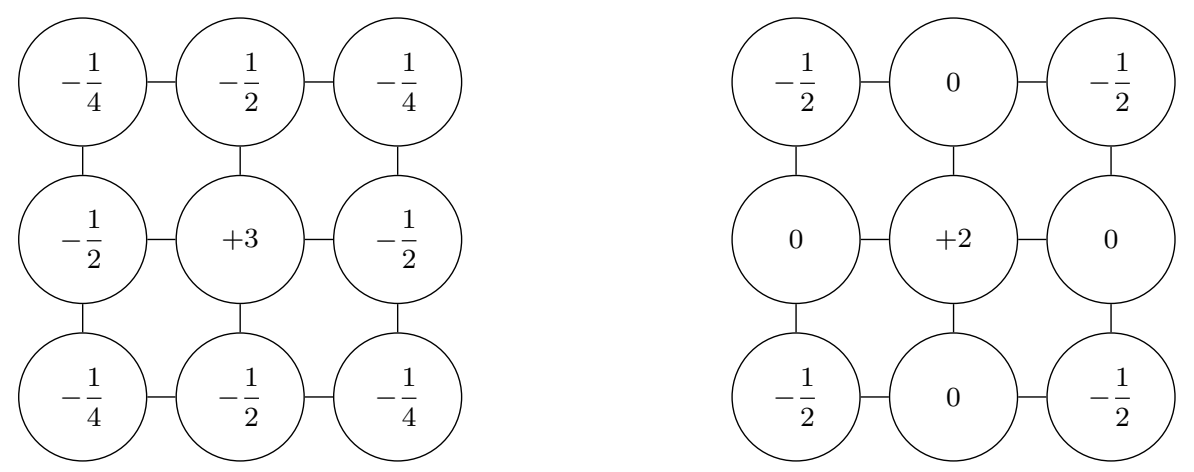

Figure 26. $\tau=1$ is a virtual element scheme. Figure 27. $\tau=0$ gives the unstable hourglass stencil.

decomposition (55).

- Square with side $a$ :

$$
\boldsymbol{U}=\frac{1}{4 a^{2}}\left[\begin{array}{cc}
2 a^{2} & 0 \\
0 & 2 a^{2}
\end{array}\right]=\left[\begin{array}{cc}
1 / 2 & 0 \\
0 & 1 / 2
\end{array}\right], \quad \int_{\Omega_{e}}|\nabla \Psi|^{2} \mathrm{~d} \boldsymbol{x}=2 / 3
$$

- Rectangle with base $a$ and height $b$ :

$$
\boldsymbol{U}=\frac{1}{4 a b}\left[\begin{array}{cc}
a^{2}+b^{2} & a^{2}-b^{2} \\
a^{2}-b^{2} & a^{2}+b^{2}
\end{array}\right], \quad \int_{\Omega_{e}}|\nabla \Psi|^{2} \mathrm{~d} \boldsymbol{x}=\frac{a^{2}+b^{2}}{3 a b} .
$$

- Rhombus with side $a$ and angle $\theta$ at vertex 1 :

$$
\begin{aligned}
\boldsymbol{U}=\frac{1}{4 a^{2} \sin \theta}\left[\begin{array}{cc}
2 a^{2}(1-\cos \theta) & 0 \\
0 & 2 a^{2}(1+\cos \theta)
\end{array}\right] & =\left[\begin{array}{cc}
\frac{1-\cos \theta}{2 \sin \theta} & 0 \\
0 & \frac{1+\cos \theta}{2 \sin \theta}
\end{array}\right], \\
\int_{\Omega_{e}}|\nabla \Psi|^{2} \mathrm{~d} \boldsymbol{x} & =\frac{2}{3 \sin \theta} .
\end{aligned}
$$

- Parallelogram with base $a$, oblique side $b$, and angle $\theta$ at vertex 1 :

$$
\boldsymbol{U}=\frac{1}{4 a b \sin \theta}\left[\begin{array}{cc}
a^{2}-2 a b \cos \theta+b^{2} & a^{2}-b^{2} \\
a^{2}-b^{2} & a^{2}+2 a b \cos \theta+b^{2}
\end{array}\right], \quad \int_{\Omega_{e}}|\nabla \Psi|^{2} \mathrm{~d} \boldsymbol{x}=\frac{a^{2}+b^{2}}{3 a b \sin \theta}
$$

Copyright (C) 2014 John Wiley \& Sons, Ltd.

Int. J. Numer. Meth. Engng 2014; 1:1-33

Prepared using nmeauth.cls 


\section{ACKNOWLEDGEMENTS}

The authors thank Franco Brezzi and Donatella Marini for their many valuable comments and suggestions. A. C. gratefully acknowledges support from the College of Science and Engineering of the University of Leicester and the support of the EPSRC (Grant EP/L022745/1). G. M. gratefully acknowledges support of the LDRD-ER Project \#20140270 "From the finite element method to the virtual element method" at the Los Alamos National Laboratory and of the DOE Office of Science Advanced Scientific Computing Research (ASCR) Program in Applied Mathematics Research. A. R. gratefully acknowledges the research support from the University of Milano-Bicocca. N. S. gratefully acknowledges the research support of the National Science Foundation through contract grant CMMI-1334783 to the University of California at Davis.

\section{REFERENCES}

[1] L. M. Taylor and D. P. Flanagan. PRONTO 2D: A two-dimensional transient solid dynamics program. Technical report, Sandia National Laboratories, Albuquerque, NM, USA, 1987.

[2] L. M. Taylor and D. P. Flanagan. PRONTO 3D: A three-dimensional transient solid dynamics program. Technical report, Sandia National Laboratories, Albuquerque, NM, USA, 1989.

[3] J. O. Hallquist. LS-DYNA3D theoretical manual. Technical report, Livermore Software Technology Corporation, Livermore, CA, USA, 1993.

[4] R. L. Taylor. FEAP $-A$ Finite Element Analysis Program VerCopyright (C) 2014 John Wiley \& Sons, Ltd.

Int. J. Numer. Meth. Engng 2014; 1:1-33

Prepared using nmeauth.cls 
sion 8.4 User Manual, 2013. University of California, Berkeley. (http://www.ce.berkeley.edu/projects/feap/manual84.pdf) [Accessed on January 10, 2014].

[5] Hibbitt, Karlsson 8 Sorensen, Inc. ABAQUS/Standard, 2004. User's Manual, Version 6.5, Pawtucket, Rhode Island, USA.

[6] S. W. Key. A finite element procedure for the large deformation dynamics response of axisymmetric solids. Computer Methods in Applied Mechanics and Engineering, 4:195$218,1978$.

[7] D. Kosloff and G. A. Frazier. Treatment of hourglass patterns in low order finite element codes. International Journal for Numerical and Analytical Methods in Geomechanics, 2:57-72, 1978.

[8] D. P. Flanagan and T. Belytschko. A uniform strain hexahedron and quadrilateral with orthogonal hourglass control. International Journal for Numerical Methods in Engineering, 17(5):679-706, 1981.

[9] W. K. Liu and T. Belytschko. Efficient linear and nonlinear heat conduction with a quadrilateral element. International Journal for Numerical Methods in Engineering, 20(5):931-948, 1984.

[10] T. Belytschko, J. S.-J. Ong, W. K. Liu, and J. M. Kennedy. Hourglass control in linear and nonlinear problems. Computer Methods in Applied Mechanics and Engineering, 43(3):251-276, 1984.

Copyright (C) 2014 John Wiley \& Sons, Ltd.

Int. J. Numer. Meth. Engng 2014; 1:1-33

Prepared using nmeauth.cls 
[11] T. Belytschko and W. E. Bachrach. Efficient implementation of quadrilaterals with high coarse-mesh accuracy. Computer Methods in Applied Mechanics and Engineering, 54(3):279-301, 1986.

[12] O.-P. Jacquotte and J. T. Oden. Analysis of hourglass instabilities and control in underintegrated finite element methods. Computer Methods in Applied Mechanics and Engineering, 44:339-363, 1984.

[13] W. K. Liu, J. S.-J. Ong, and R. A. Uras. Finite element stabilization matrices a unification approach. Computer Methods in Applied Mechanics and Engineering, 53(1):13-46, 1985 .

[14] B. C. Koh and N. Kikuchi. New improved hourglass control for bilinear and trilinear elements in anisotropic linear elasticity. Computer Methods in Applied Mechanics and Engineering, 65:1-46, 1987.

[15] T. B. Belytschko, W. K. Liu, and B. Moran. Nonlinear Finite Elements for Continua and Structures. John Wiley and Sons, New York, 2000.

[16] L. Beirão da Veiga, F. Brezzi, A. Cangiani, G. Manzini, L. D. Marini, and A. Russo. Basic principles of virtual element methods. Mathematical Models $\mathscr{E}$ Methods in Applied Sciences, 23(1):199-214, 2014.

[17] G. L. Goudreau and J. O. Hallquist. Recent developments in large-scale finiteelement Lagrangian hydrocode technology. Computer Methods in Applied Mechanics and Engineering, 33(1-3):725-757, 1982.

Copyright (c) 2014 John Wiley \& Sons, Ltd.

Int. J. Numer. Meth. Engng 2014; 1:1-33

Prepared using nmeauth.cls 
[18] L. Beirão da Veiga, F. Brezzi, L.D. Marini, and A. Russo. The hitchhiker's guide to the virtual element method. Mathematical Models 83 Methods in Applied Sciences, 24(8):1541$1573,2014$.

[19] G. Manzini, A. Russo, and N. Sukumar. New perspectives on polygonal and polyhedral finite element methods. Mathematical Models 83 Methods in Applied Sciences, 24(8):16651699, 2014.

[20] C. Talischi and G. H. Paulino. Addressing integration error for polygonal finite element through polynomial projections: A patch test connection. Mathematical Models \& Methods in Applied Sciences, 24(8):1701-1727, 2014.

[21] K. Lipnikov, G. Manzini, and M. Shashkov. Mimetic finite difference method. Journal of Computational Physics, 257 - Part B:1163-1227, 2014.

[22] L. Beirão da Veiga, K. Lipnikov, and G. Manzini. The Mimetic Finite Difference Method, volume 11 of Modeling, Simulations and Applications. Springer-Verlag, New York, I edition, 2013.

[23] B. Ahmad, A. Alsaedi, F. Brezzi, L.D. Marini, and A. Russo. Equivalent projectors for virtual element methods. Computer $\mathcal{E}$ Mathematics with Applications, 66(3):376-391, 2013.

[24] P. Hansbo. A new approach to quadrature for finite elements incorporating hourglass control as a special case. Computer Methods in Applied Mechanics and Engineering, 158(3):301-309, 1998.

Copyright (C) 2014 John Wiley \& Sons, Ltd.

Int. J. Numer. Meth. Engng 2014; 1:1-33

Prepared using nmeauth.cls 
[25] T. Belytschko. Correction of article by D. P. Flanagan and T. Belytschko. International Journal for Numerical Methods in Engineering, 19(3):467-468, 1983.

[26] F. Brezzi and L. D. Marini. Virtual element methods for plate bending problems. Computer Methods in Applied Mechanics and Engineering, 253:455-462, 2013.

[27] L. Beirão da Veiga and G. Manzini. A virtual element method with arbitrary regularity. IMA Journal on Numerical Analysis, 34(2):759-781, 2014.

[28] L. Beirão da Veiga, F. Brezzi, and L. D. Marini. Virtual elements for linear elasticity problems. SIAM Journal on Numerical Analysis, 51(2):794-812, 2013.

[29] A. Gain, C. Talischi, and G. H. Paulino. On the virtual element method for threedimensional linear elasticity problems on arbitrary polyhedral meshes. Computer Methods in Applied Mechanics and Engineering, 282:132-160, 2014.

Copyright (C) 2014 John Wiley \& Sons, Ltd.

Int. J. Numer. Meth. Engng 2014; 1:1-33

Prepared using nmeauth.cls 